Ferlizza E., Isani G., Dondi F., Andreani G., Vasylyeva K., Bellei E., Almeida A.M., Matzapetakis M. 2020. Urinary proteome and metabolome in dogs (Canis lupus familiaris): The effect of chronic kidney disease. Journal of Proteomics 222: 103795. https://doi.org/10.1016/j.jprot.2020.103795

\title{
Urinary proteome and metabolome in dogs (Canis lupus familiaris): The effect of chronic kidney disease
}

Author links open overlay

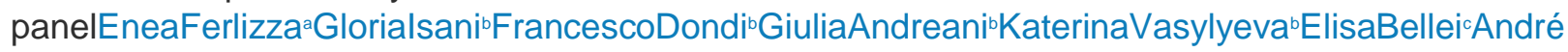
M.AlmeidadManolisMatzapetakis ${ }^{\circ}$

Show more

Add to Mendeley

Share

Cite

https://doi.org/10.1016/j.jprot.2020.103795Get rights and content

\section{Highlights}

Urinary proteome and metabolome were studied in healthy and CKD dogs.

$\cdot$

Proteomics highlighted a decrease of uromodulin and an increase of albumin.

$\bullet$

${ }^{1} \mathrm{H}$ NMR evidenced 17 metabolites significantly different between healthy and CKD dogs. 
Proteomics and metabolomics successfully suggested putative biomarkers for CKD.

\section{Abstract}

Chronic kidney disease (CKD) is a progressive and irreversible disease. Although urine is an ideal biological sample for proteomics and metabolomics studies, sensitive and specific biomarkers are currently lacking in dogs. This study characterised dog urine proteome and metabolome aiming to identify and possibly quantify putative biomarkers of CKD in dogs. Twenty-two healthy dogs and 28 dogs with spontaneous CKD were selected and urine samples were collected. Urinary proteome was separated by SDS-PAGE and analysed by mass spectrometry, while urinary metabolome was analysed in protein-depleted samples by $1 \mathrm{D}{ }^{1} \mathrm{H}$ NMR spectra. The most abundant proteins in urine samples from healthy dogs were uromodulin, albumin and, in entire male dogs, arginine esterase. In urine samples from CKD dogs, the concentrations of uromodulin and albumin were significantly lower and higher, respectively, than in healthy dogs. In addition, these samples were characterised by a more complex protein pattern indicating mixed glomerular (protein bands $\geq 65 \mathrm{kDa}$ ) and tubular (protein bands $<65 \mathrm{kDa}$ ) proteinuria. Urine spectra acquired by NMR allowed the identification of 86 metabolites in healthy dogs, belonging to 49 different pathways mainly involved in amino acid metabolism, purine and aminoacyl-tRNA biosynthesis or tricarboxylic acid cycle. Seventeen metabolites showed significantly different concentrations when comparing healthy and CKD dogs. In particular, carnosine, trigonelline, and cis-aconitate, might be suggested as putative biomarkers of CKD in dogs.

\section{Significance}

Urine is an ideal biological sample, however few proteomics and metabolomics studies investigated this fluid in dogs and in the context of CKD (chronic kidney disease). In this research, applying a multi-omics approach, new insights were gained regarding the molecular changes triggered by this disease in canine urinary proteome and metabolome. In particular, the involvement of the tubular component was 
highlighted, suggesting uromodulin, trigonelline and carnosine as possible biomarkers of CKD in dogs.

\section{Graphical abstract}
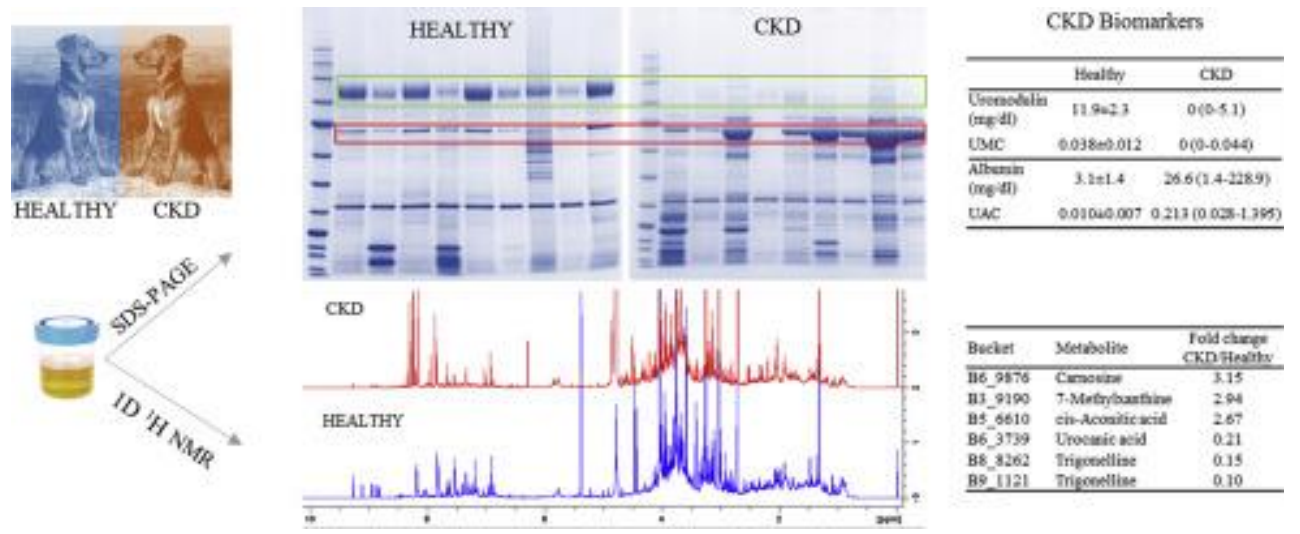

1. Download: Download high-res image (348KB)

2. Download: Download full-size image

- Previous article in issue

- Next article in issue

\section{Introduction}

Chronic kidney disease (CKD) is a progressive and irreversible disease characterised by the presence of structural or functional abnormalities in one or both kidneys over a period of three months or longer [1]. CKD is one of the most common renal diseases in dogs with an estimated prevalence varying from 0.5 to $3.64 \%$ depending on the inclusion criteria of the cases [[1], [2], [3]]. Early diagnosis of CKD may hinder the disease progression and improve patient quality of life. International Renal Interest Society (IRIS) guidelines for staging and treatment of CKD help clinicians to correctly classify patients and establish the best therapies [4]. Nonetheless, sensitive and specific biomarkers for early detection and monitoring of CKD in dogs are currently lacking. The gold standard to evaluate the renal function is the determination of the glomerular filtration rate (GFR); however, this value does not provide information on CKD aetiology and the available methods for its estimation are difficult to be applied in the routine clinical practice $[\underline{5}, 6]$. Renal biopsy is considered the gold standard for determining the type of renal damage, but it is an invasive 
procedure and not always feasible [7]. Therefore, the assessment of the kidney function is currently based on conventional blood (serum creatinine or urea) and urine (proteinuria and specific gravity) clinicopathological variables, whose alterations are usual findings of CKD, but have limitations when used as early indicators of the disease [7]. For these reasons, other sensitive and specific biomarkers measurable in non- or minimally invasive biological samples are required in clinical practice to identify early renal damage in dogs.

Over the last years, significant efforts have been made in veterinary medicine to apply proteomics to search for new biomarkers or for validating detection methods for proteins already considered as potential early indicators of kidney disease in dogs and cats [[8], [9], [10], [11], [12], [13], [14], [15], [16], [17]]. However, proteins are only some of the molecular species present in urine and a broader approach with the aid of metabolomics can offer additional clinical information.

Metabolomics enables the assessment of a broad range of endogenous and exogenous small molecular mass metabolites, potentially useful to investigate the physiologic status and the pathogenesis of the diseases, and to discover new biomarkers of altered biochemical pathways [[18], [19], [20], [21]]. Metabolites are in general not specific for a single metabolic pathway and in most cases different biochemical reactions contribute to the production of the same metabolite; this peculiarity offers the opportunity to obtain a more comprehensive insight into the complexity of a biological sample. In human medicine, metabolomics was extensively applied to urine to analyse the healthy metabolome [22] and to search for small molecules as potential biomarkers of different diseases, such as immune-mediated inflammatory diseases [23], different cancers [[24], [25], [26]], and renal diseases [19,[27], [28], [29], [30]]. However, in veterinary medicine, the application of metabolomics techniques to urine is still limited [[31], [32], [33], [34], [35]].

Owing to the metabolic and protein complexity of urine, the aim of this work was to combine the analytical power of proteomics and metabolomics to obtain a more comprehensive characterisation of the urine in healthy dogs and to compare it with the urine of CKD patients with our ultimate goal to suggest new biomarkers of CKD in the canine species.

\section{Materials and methods}




\subsection{Animal selection and sample collection}

The present study was performed on urine samples collected at the Veterinary Teaching Hospital of the University of Bologna from owned dogs. The dogs were divided into two experimental groups and specimens considered as biological replicates. Upon arrival, all dogs were subjected to physical examination and routine laboratory tests, including complete blood count, serum chemistry and complete urinalysis with urine protein to creatinine ratio (UPC).

Blood samples were collected by venipuncture using a vacuum collection system (Vacutest Kima, Arzergrande, Italy) after at least a 12-h fasting period. Blood samples were processed within one hour after collection. Serum samples were collected in tubes with clot activator (Vacutest Kima, Arzergrande, Italy), centrifuged at $3000 \mathrm{~g}$ for $10 \mathrm{~min}$ and analysed in an automated chemistry analyser (AU 480, Olympus/Beckman Coulter, Atlanta, GE, USA).

Urine samples were collected by ultrasound-guided cystocentesis. All urine specimens were processed on a routine basis and evaluated in our laboratory within $24 \mathrm{~h}$ after collection. In particular, urinalysis consisted in macroscopic examination, urine specific gravity (USG) measured by manual refractometer (American Optical, Buffalo, New York), urine dipstick test (Combur10Test, Roche Diagnostic, Mannheim, Germany) applied on an automated reader (Urisys 1100, Roche Diagnostic, Mannheim, Germany) and microscopic sediment evaluation. Urine sediment was obtained after centrifugation at $500 \mathrm{~g}$ for $10 \mathrm{~min}$. Urine supernatants were immediately analysed (dipstick examination), divided in aliquots and stored at $-80{ }^{\circ} \mathrm{C}$ for the subsequent proteomics and metabolomics analysis. Urine chemistry was performed on a refrigerated $\left(+4{ }^{\circ} \mathrm{C}\right)$ aliquot if performed within $24 \mathrm{~h}$ after the sample processing or on an aliquot kept frozen at $-20{ }^{\circ} \mathrm{C}$ for a maximum of 7 days. Dogs were considered healthy or diseased on the basis of history, clinical signs and the results of the above-mentioned routine laboratory tests. The control group included 22 healthy dogs presented at the hospital as blood donors. The 22 healthy dogs were 10 males ( 3 castrated) and 12 females ( 7 spayed) with an average age of $37 \pm 20$ months. Mixed-breed dogs were 7/22 (32\%), while purebred dogs were 15/22 (68\%) (3 Galgo Espanol, 3 Labrador Retriever, 2 Australian Shepherd, 1 Great Dane, 
1 Border Collie, 1 Flat-Coated Retriever, 1 German Shepherd, 1 Miniature Pinscher, 1 Boxer, 1 Weimaraner). The diseased group included 28 dogs affected by naturally occurring CKD. The 28 CKD dogs were 14 males ( 5 castrated) and 14 females (9 spayed) with a mean age of $111 \pm 61$ months. Mixed-breed dogs were 10/28 (36\%), while purebred dogs were 18/28 (64\%) (3 Jack Russell Terrier, 2 Chinese Shar-Pei, 2 Labrador Retriever, 2 Cavalier King Charles spaniel, 1 Beagle, 1 Bernese mountain dog, 1 Boxer, 1 German Shepherd, 1 English Springer spaniel, 1 American Staffordshire terrier, 1 Bull terrier, 1 German shorthaired pointer, 1 Lagotto Romagnolo). The diagnosis of CKD was based on history, clinical signs, clinicopathological and imaging results, according to the literature [프느. In particular, the presence of clinical findings, abdominal imaging results and (a) persistent pathologic renal proteinuria based on the UPC (UPC > 0.5), assessed and confirmed over a one-month period, and/or (b) serum creatinine (sCrea) concentration $\geq 1.40 \mathrm{mg} / \mathrm{dL}$ and/or (c) urine specific gravity (USG) $<1.030$ were considered diagnostic. The IRIS CKD guidelines were used to subsequently stage CKD dogs [4]. Basing on serum creatinine, 8 dogs were classified with CKD stage 1, 6 with stage 2, 9 with stage 3 and 5 with stage 4 . On the basis of UPC, 4 dogs were non-proteinuric (UPC < 0.2), 6 dogs were borderline proteinuric (UPC 0.2-0.5) and 18 were proteinuric (UPC > 0.5).

The study was conducted according to the EU Directive 2010/63/EU for animal experiments and approved by the Institutional Scientific Ethical Committee of the University of Bologna for animal testing.

\subsection{Urine protein to creatinine ratio}

Five $\mathrm{mL}$ of urine were collected from each animal by ultrasound-guided cystocentesis. After centrifugation at $500 \mathrm{~g}$ for $10 \mathrm{~min}$, urine total proteins and creatinine were measured using commercial kits (Urinary/CSF Protein, OSR6170, and Creatinine OSR6178, Olympus/Beckman Coulter, Atlanta, GE, USA) on an automated chemistry analyser (AU 480, Olympus/Beckman Coulter, Atlanta, GE, USA). The UPC was calculated with the following formula: UPC $=$ urine protein $(\mathrm{mg} / \mathrm{dL}) /$ urine creatinine $(\mathrm{mg} / \mathrm{dL})$.

\subsection{SDS-PAGE and protein identification}


Urine proteins were separated using an electrophoresis system (NuPAGE, Thermo Fisher Scientific, Waltham, MA, USA) as previously described [묘, $\underline{6}]$. Briefly, three to five $\mu \mathrm{g}$ of protein were loaded on 4-12\% polyacrylamide gel in MOPS buffer with SDS (Thermo Fisher Scientific, Waltham, MA, USA). The gels were stained with Coomassie brilliant blue (PageBlu protein staining solution; Thermo Fisher Scientific, Waltham, MA, USA) compatible with mass spectrometry analysis. After staining, each gel was digitalised (ChemidocMP, BioRad, Hercules, California, USA) and the pherograms were obtained using a commercial software (ImageLab, BioRad, Hercules, California, USA). The bands at 100, 67 and $18 \mathrm{kDa}$ were cut and identified by electrospray ionization quadrupole time-of-flight mass spectrometry (ESI-QTOF/MS) as previously reported $[\underline{8}, \underline{36}]$.

To quantify the bands at $100 \mathrm{kDa}$ and $67 \mathrm{kDa}$, on each sample, one $\mu \mathrm{g}$ of protein, obtained from a solution containing $1 \mu \mathrm{g} / \mu \mathrm{L}$ of lactate dehydrogenase (LDH), (SigmaAldrich/Merck KGaA, Darmstadt, Germany) was added as internal standard of quantity. The ImageLab software estimated the volume of each protein band based on pixel density within the band boundaries in the digital image. The volume of the band of interest was then compared to the internal standard (LDH) of the corresponding lane and the concentration was calculated as follows:Xmg/dL=Vband/VLDH/ $\mu$ Lsample $* 100$. $\mathrm{X}=$ concentration of the protein at $100 \mathrm{kDa}$ or at $67 \mathrm{kDa}$.

$\mathrm{V}_{\text {band }}=$ volume of the band at $100 \mathrm{kDa}$, or at $67 \mathrm{kDa}$ determined by the software. $\mathrm{V}_{\mathrm{LDH}}=$ volume of the band of the internal standard (LDH) determined by the software. $\mu \mathrm{L}_{\text {sample }}=\mu \mathrm{L}$ of the sample loaded in the gel.

Subsequently, the respective ratios with urine creatinine (uromodulin $[\mathrm{mg} / \mathrm{dL}]$ : creatinine $[\mathrm{mg} / \mathrm{dL}]$, UMC; albumin $[\mathrm{mg} / \mathrm{dL}]$ : creatinine $[\mathrm{mg} / \mathrm{dL}], \mathrm{UAC})$ were calculated.

\subsection{NMR sample preparation}

Urine metabolites were extracted for NMR as follows: $500 \mu \mathrm{L}$ of urine supernatants were mixed with $550 \mu \mathrm{L}$ of chloroform and $550 \mu \mathrm{L}$ of methanol, vortexed for $1 \mathrm{~min}$, left to rest for $15 \mathrm{~min}$ at $+4{ }^{\circ} \mathrm{C}$ and centrifuged at $12000 \mathrm{~g}$ for $15 \mathrm{~min}$ at room temperature. Nine hundred $\mu \mathrm{L}$ of the upper phase (urine/methanol) were dried in a 
vacuum centrifuge (SpeedVac, Thermo Fischer Scientific, Waltham, MA, USA) overnight at $30{ }^{\circ} \mathrm{C}$. The resulting pellets were suspended with $200 \mu \mathrm{L}$ of phosphate buffer (PB, $240 \mathrm{mM} \mathrm{pH} 7.4$ in $\mathrm{D}_{2} \mathrm{O}$ with trimethylsilylpropanoic acid [TSP] and sodium azide $\left.\left[\mathrm{NaN}_{3}\right]\right)$ and $400 \mu \mathrm{L}$ of $\mathrm{D}_{2} \mathrm{O}$ to a final concentration of $80 \mathrm{mM} \mathrm{PB}$, $0.087 \mathrm{mM}$ TSP and $0.022 \%(\mathrm{v} / \mathrm{v}) \mathrm{NaN}_{3}$. Samples were vortexed for $1 \mathrm{~min}$, centrifuged at $12,000 \mathrm{~g}$ for $1 \mathrm{~min}$ and $560 \mu \mathrm{L}$ transferred into a $5 \mathrm{~mm}$ NMR tubes.

\subsection{NMR acquisition}

NMR spectroscopy was conducted on an $800 \mathrm{MHz}$ spectrometer with a triple resonance HCN Z-gradient probe, at $298 \mathrm{~K}$ (Bruker AvanceII+, Ettlingen, Germany). Acquisition and processing were carried out using standard software (Topsin 3.2, Bruker Biospin, Billerica, MA, USA). One dimensional 'H NMR spectra with CarrPurcell-Meiboom-Gill (CPMG) filter to attenuate signals from macromolecules were acquired using a standard vendor pulse sequence (cpmgpr1d). Spectra were acquired at $25^{\circ} \mathrm{C}$, with a $20 \mathrm{ppm}$ spectral width, spin lock duration of $78.72 \mathrm{~ms}$, presaturation for $4 \mathrm{~s}$ using $20 \mu \mathrm{W}$ and acquisition time of $2 \mathrm{~s}$. A total of 16 dummy scans and 128 scans were acquired for each sample. All spectra were processed with an exponential window function with $1 \mathrm{~Hz}$ line broadening and automated phasing and baseline correction. For the chemometric analysis, the processed data were further processed in the "nmrprocflow" platform [37]. Bins were obtained using manually curated, intelligent binning after referencing, baseline correction, water signal removal and peak alignment. For selected samples, additional homonuclear and heteronuclear spectra ( ${ }^{1} \mathrm{H} J$-resolved, ${ }^{1} \mathrm{H}_{-}{ }^{1} \mathrm{H}$ COSY, and $\left.{ }^{1} \mathrm{H}_{-}{ }^{13} \mathrm{C} \mathrm{HSQC}\right)$ were also collected to assist with compound identification.

\subsection{Metabolite annotation and identification}

The bins obtained from the "nmrprocflow" platform [37] were annotated with the help of database assisted spectral decomposition using commercial software (Chenomx 8.2 NMR Suit, Edmonton, Alberta, Canada) and the internal reference library (Version 10) as well as the Biological Magnetic Resonance Data Bank (BMRB, http://www.bmrb.wisc.edu) reference spectra for compounds absent in the internal reference library. Buckets were attributed to multiple metabolites where peaks 
were found to overlap. Pathway analysis module of a free web-based analytical platform (Metaboanalyst 4.0, www.metaboanalyst.ca), that used the high-quality Kyoto encyclopaedia of genes and genomes (KEGG) metabolic pathways as the backend knowledgebase, was used to search for the metabolic pathways.

\subsection{Statistical analysis}

Serum and urine chemistry data were analysed with statistical software ( $\mathrm{R}$ version 3.4.4). Normal distribution was tested graphically and by Shapiro-Wilk normality test, and data were expressed as mean \pm standard deviation (SD) or median (range; minimum - maximum value) if normally or non-normally distributed, respectively. Variables were compared between healthy $(N=22)$ and CKD $(N=28)$ dogs using the Student $t$-test or the Mann-Whitney $U$ test depending on their distribution, assuming $P<.05$ as a significant probability. The Kruskal-Wallis rank sum test was applied to evaluate differences among healthy and CKD stages (stages $1-4$, basing on serum creatinine and according to the IRIS guidelines [4]) and adjusted $P$-values lower than 0.05 were considered statistically significant.

For metabolomics statistical analysis, processed spectra were aligned, baseline corrected and divided into 397 variable width spectral regions or 'buckets' with the intensity of each bucket divided by the bucket width. To identify the signals differentially present in the two groups, the buckets were loaded into a web-based platform (Metaboanalyst 4.0, www.metaboanalyst.ca) which uses the R package of statistical computing software [38]. For multivariate analysis, buckets were scaled by auto-scaling (mean-centred and divided by the standard deviation of each variable) while, for univariate analysis, and in order to remove the influences attributed to muscle mass and urine concentration, the bucket intensities were normalised to the peak of creatinine (bucket $3.0360 \mathrm{ppm}$ ). Both univariate and multivariate statistics were employed. $t$-test and fold change analysis were used to identify the buckets with differential presence, while the list was supplemented with the use of unsupervised principal components analysis (PCA) and supervised partial least squares discriminant analysis (PLS-DA). Both PCA and PLS-DA can identify signals (buckets) whose importance becomes significant via correlated variance. In addition, PCA provides a global view of the differentiability between the two experimental conditions and the 
groups of observables that are mostly responsible. In contrast, PLS-DA, since it is a supervised method, highlights the variables most responsible for the differences between groups as previously used in other metabolomics approaches $[\underline{39}, \underline{40}]$. The small sample size that is typical in such studies and the inherent large number of variables obtained may affect the consistency of the multivariate analysis used. To evaluate the consistency of the results, the software performs a number of tests and reports the parameters $\mathrm{Q}^{2}$ and $\mathrm{R}^{2}$ as quality parameters of the models. $\mathrm{Q}^{2}$ indicates the predictive ability of the model, while $\mathrm{R}^{2}$ is the indicator of the suitability of the fit. For PLSDA Q ${ }^{2}>0.6$ were selected as acceptable models. Variable importance in projection (VIP) scores greater than 1 and $t$-test with a $P$ value $<.05$ were used to identify metabolites as differentially expressed.

\section{Results}

\subsection{Clinical data}

Mean clinical data, serum and urine biochemistry of healthy and CKD dogs are reported in Table 1, while the results for each dog are reported in Supplement Table 1.

Table 1. Clinical data for healthy and CKD dogs. Data are reported as mean \pm SD or median (range) depending on normal or non-normal distribution, respectively.

\begin{tabular}{|c|c|c|c|c|c|}
\hline Signalment & Healthy ( $N=22)$ & CKD (N = 28) & & & $P$ \\
\hline Age in months & $37 \pm 20$ & $112 \pm 61$ & & & $<0.0001$ \\
\hline Female $\mathrm{n}$ (entire/neutered) & $12(5 / 7)$ & $14(5 / 9)$ & & & \\
\hline Male n (entire/neutered) & $10(7 / 3)$ & $14(9 / 5)$ & & & \\
\hline Serum biochemistry & Healthy & CKD & $\mathbf{N}(\%)$ CKD < / > RI & $\mathbf{R I}$ & \\
\hline Total Proteins (g/dL) & $6.4 \pm 0.4$ & $6.0 \pm 0.8$ & $5(17.9)</ 1(3.6)>$ & $5.6-7.3$ & 0.109 \\
\hline Albumin (g/dL) & $3.4 \pm 0.3$ & $3.0(1.1-3.8)$ & $12(42.9)<$ & $2.8-3.9$ & $<0.0001$ \\
\hline Creatinine (mg/dL) & $1.1 \pm 0.2$ & $2.0(0.6-9.8)$ & $1(3.6)</ 20(71.4)>$ & $0.8-1.4$ & $<0.0001$ \\
\hline Urea $(\mathrm{mg} / \mathrm{dL})$ & $33 \pm 8$ & $110(17-519)$ & $22(78.6)>$ & $17-48$ & $<0.0001$ \\
\hline
\end{tabular}




\begin{tabular}{|c|c|c|c|c|c|}
\hline Signalment & Healthy $(N=22)$ & CKD (N = 28) & & & $P$ \\
\hline Phosphorus (mg/dL) & $4.6 \pm 0.9$ & $4.9(2.6-14.1)$ & $11(39.3)>$ & $2.7-5.4$ & 0.056 \\
\hline Urine biochemistry & Healthy & CKD & $\mathbf{N}(\%)$ CKD < / > RI & $\mathbf{R I}$ & \\
\hline UPC & $0.07(0.04-0.19)$ & $0.78(0.09-12.8)$ & $18(64.3)>$ & $<0.5$ & $<0.0001$ \\
\hline USG & $1.052(1.034-1.064)$ & $1.014(1.006-1.062)$ & $27(96.4)<$ & $>1.030$ & $<0.0001$ \\
\hline IRIS Stage & & $\mathbf{N}(\%)$ & & $\mathbf{R} \mathbf{I}$ & \\
\hline I & & $8(28.6)$ & & $<1.4$ & \\
\hline ॥ & & $6(21.4)$ & & $1.4-2.0$ & \\
\hline III & & $9(32.1)$ & & $2.1-5.0$ & \\
\hline IV & & $5(17.8)$ & & $>5.0$ & \\
\hline
\end{tabular}

RI, reference intervals; N, number of samples; UPC, urine protein to creatinine ratio; USG, urine specific gravity;

a

Considered as adequate USG in dogs.

CKD dogs were significantly older $(P<.0001)$, had significantly higher concentration of serum creatinine $(P<.0001)$, urea $(P<.0001)$ and UPC $(P<.0001)$, while USG $(P<.0001)$ was significantly lower than in the healthy dogs. CKD patients were also staged according to serum creatinine concentration following IRIS guidelines [4] and the differences of UPC and USG were evaluated. USG was significantly lower in each CKD stage group than in the healthy dogs $(P<.01)$, and samples classified as CKD stage 1 had higher USG than those classified as Stage $3(P=.016)$ and $4(P=.007)$. UPC was significantly higher in each CKD stage group than in healthy dogs $(P<.05)$, however, no significant differences were found among CKD stages.

\subsection{SDS-PAGE proteomics analysis}


Representative gels and pherograms from healthy and CKD dog urines are reported in Fig. 1.

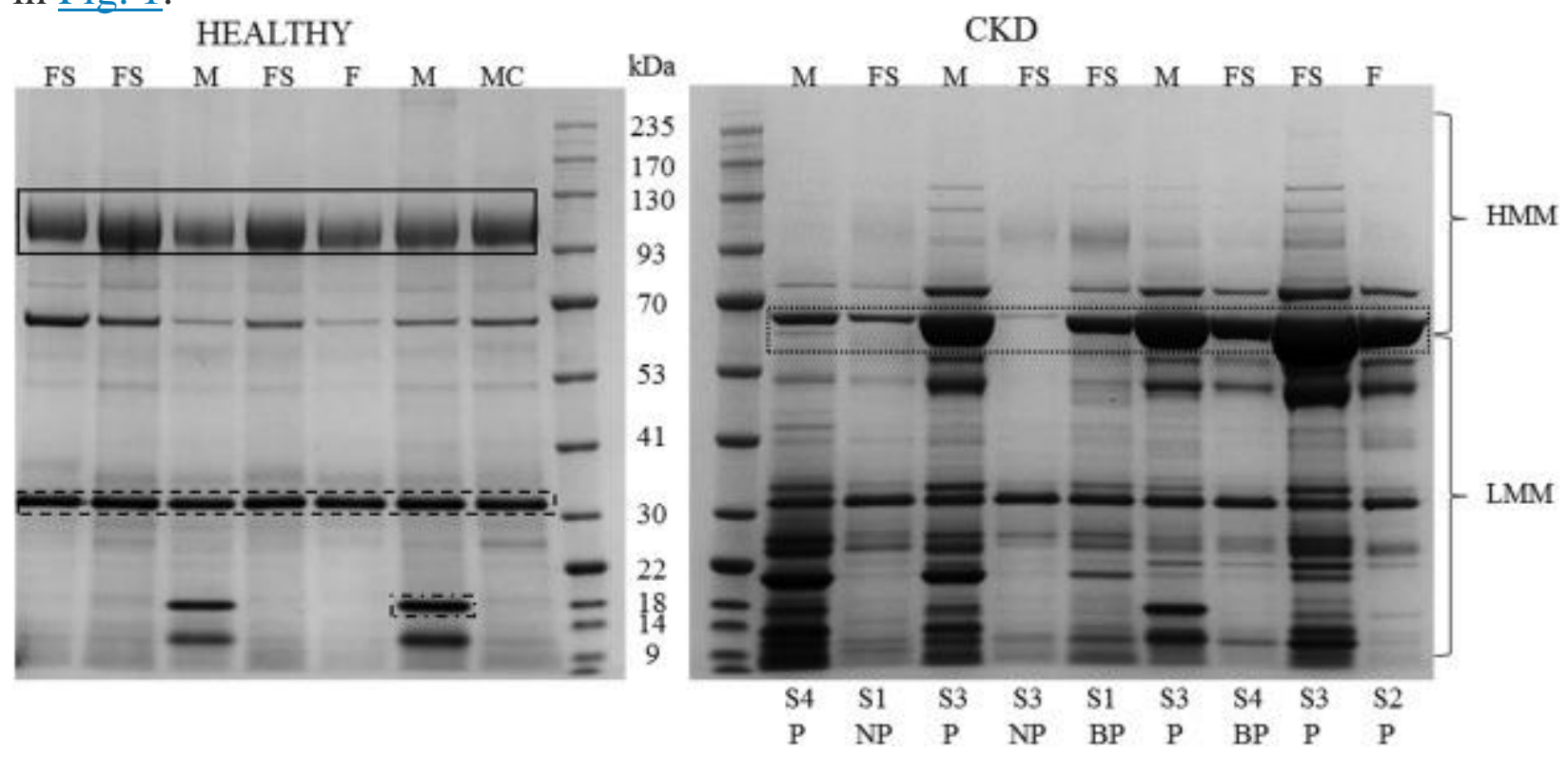

1. Download : Download high-res image (557KB)

2. Download: Download full-size image

Fig. 1. Representative SDS-PAGE gels of urine samples from healthy and CKD dogs. Black continuous box indicates uromodulin $(103 \mathrm{kDa})$; black dotted box indicates albumin $(67 \mathrm{kDa})$; black dashed box indicates the internal standard of quantity $(1 \mu \mathrm{g})$; black dashed and dotted box indicates arginine esterase (18 kDa). M, male; MC, male castrated; F, female; FS, female spayed. LMM, low molecular mass $(\mathrm{kDa}<67 \mathrm{kDa})$; HMM high molecular mass $(\mathrm{kDa}>67 \mathrm{kDa})$. S1-4 under each lane indicate the CKD stage of the patient according to IRIS guideline. NP (non-proteinuric, UPC < 0.2), BP (borderline proteinuric, UPC 0.2-0.5) or P (proteinuric, UPC > 0.5) under each lane indicate the classification of proteinuria according to IRIS guideline.

Urine samples from the healthy group presented similar profiles characterised by the presence of three most abundant bands at apparent molecular mass (MM) of 103, 80 and $67 \mathrm{kDa}$, respectively. The bands at 103 and $67 \mathrm{kDa}$ were identified by mass spectrometry as uromodulin and albumin, respectively (Table 2). Moreover, most of the samples presented other three to five low abundance bands at apparent MM between 55 and $14 \mathrm{kDa}$ and two bands at $\mathrm{MM}<14 \mathrm{kDa}$. In addition, urine samples from entire males presented other two evident bands at apparent MM of 18 and $12 \mathrm{kDa}$. The band at $18 \mathrm{kDa}$ was identified as arginine esterase (Table 2).

Table 2. Proteins identified in dog urine by mass spectrometry. 


\begin{tabular}{|c|c|c|c|c|c|c|c|}
\hline Protein name & Protein entry name & $\mathrm{MM}(\mathrm{kDa})$ & Score $\_$ & Pept $\stackrel{d}{ }$ & Sign Pept $\mathrm{e}$ & Seq & Sign seq \\
\hline Uromodulin & UROM_CANFA & 73 & 2298 & 138 & 113 & 15 & 13 \\
\hline Albumin & ALBU_CANFA & 69 & 5802 & 470 & 321 & 44 & 39 \\
\hline Arginine esterase & ESTA_CANFA & 29 & 532 & 111 & 52 & 10 & 9 \\
\hline
\end{tabular}

Protein entry name from UniProt knowledge database.

b

Theoretical protein molecular mass.

C

The highest scores obtained with Mascot search engine.

d

Peptides: total number of peptides matching the identified proteins.

e

Significant peptides: total number of significant peptides matching the identified proteins.

$\mathrm{f}$

Sequence: total number of distinct sequences matching the identified proteins.

g

Significant sequences: total number of significant distinct sequences matching the identified proteins.

CKD samples presented different and more variable electrophoretic profiles. The disappearance of uromodulin and/or the increase of intensity of albumin and of the band at $80 \mathrm{kDa}$ were clearly evident in all the analysed samples. The increase in number and intensity of the bands at high $(>67 \mathrm{kDa})$ and low $(<67 \mathrm{kDa}) \mathrm{MM}$ was also evidenced. Particularly, two samples presented an increase in number and intensity of 
the bands at high (>67 kDa) MM only, nine samples showed an increase in number and intensity of the bands at low ( $<67 \mathrm{kDa}) \mathrm{MM}$ only, while the remaining 17 samples presented an increase in number and intensity of the bands at both high and low MM. Additionally, in 12 samples (Fig. 1; Lanes 2, 4, 6, 9) was evidenced a band at $21 \mathrm{kDa}$ that was not present in healthy samples.

Concentrations of uromodulin and albumin and their ratio with creatinine (UMC and UAC) are reported in Table 3. Urine samples from healthy dogs presented a low amount of albumin $(3.1 \pm 1.4 \mathrm{mg} / \mathrm{dL})$ and a high amount of uromodulin $(11.9 \pm 2.3 \mathrm{mg} / \mathrm{dL})$. CKD dogs presented a significantly higher concentration of albumin $(P=.0025)$ and UAC value $(P=.0002)$ and a significantly lower concentration of uromodulin $(P<.0001)$ and UMC value $(P=.0044)$, compared to healthy animals.

Table 3. Data for albumin and uromodulin quantification by SDS-PAGE. Data are reported as mean \pm SD or median (range) depending on normal or non-normal distribution, respectively.

\section{Healthy}

$3.1 \pm 1.4$

$0.010 \pm 0.007$

$11.9 \pm 2.3$

$0.038 \pm 0.012$
CKD

$26.6(1.4-228.9)$

$0.213(0.028-1.395)$

$0(0-5.1)$

$0(0-0.044)$
0.0025

0.0002

$<0.0001$

0.0044

\subsection{Metabolites annotation and identification}

Representative NMR spectra from healthy and CKD dog urine samples are reported in Fig. 2. 


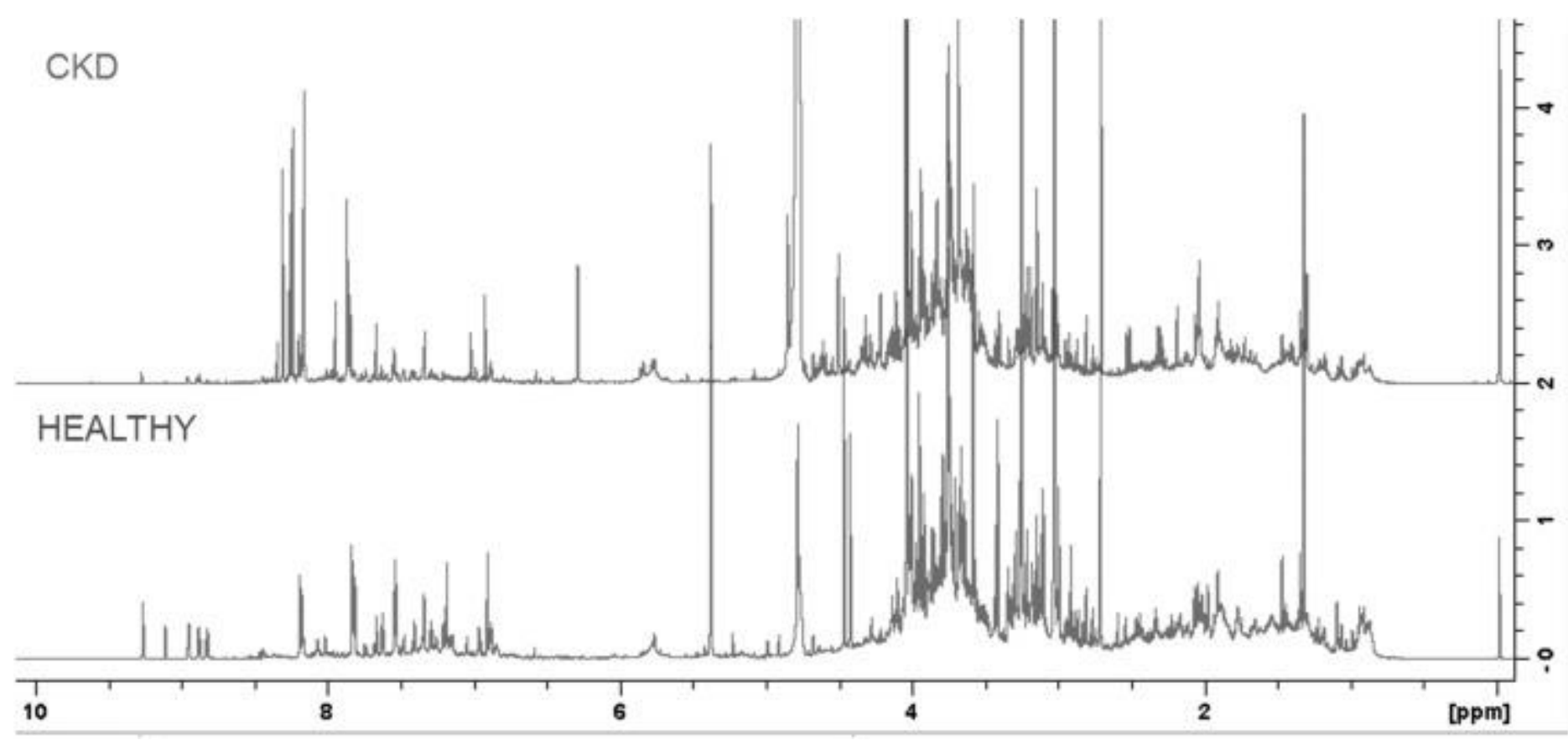

1. Download: Download high-res image (219KB)

2. Download: Download full-size image

Fig. 2. Representative NMR spectra of urine samples collected from healthy and CKD dogs.

An overview of the NMR spectra of samples from healthy dogs evidenced similar profiles, while the urine from CKD patients showed more variable spectra and differences in metabolite abundance. From the 397 buckets, 86 metabolites were identified in healthy samples, with different biological functions and belonging to different pathways. An entire spectrum of the urine of an healthy dog with the assigned metabolites is reported in Fig. 3. The five most abundant metabolites were creatinine, urea, taurine, lactate and 1-methylnicotinamide, while the list of all the identified metabolites is reported in Table 4. After MetaboAnalyst pathway analysis, metabolites were shown as belonging to 49 different pathways, and 23 of these pathways were represented by at least 3 different metabolites. The most represented pathways are mainly involved in amino acid metabolism, purine and aminoacyl-tRNA biosynthesis and tricarboxylic acid cycle (Table 5). In particular, 10 metabolites belonged to glycine, serine and threonine metabolism and aminoacyl-tRNA biosynthesis, while 8 metabolites were involved in phenylalanine metabolism and purine metabolism. 


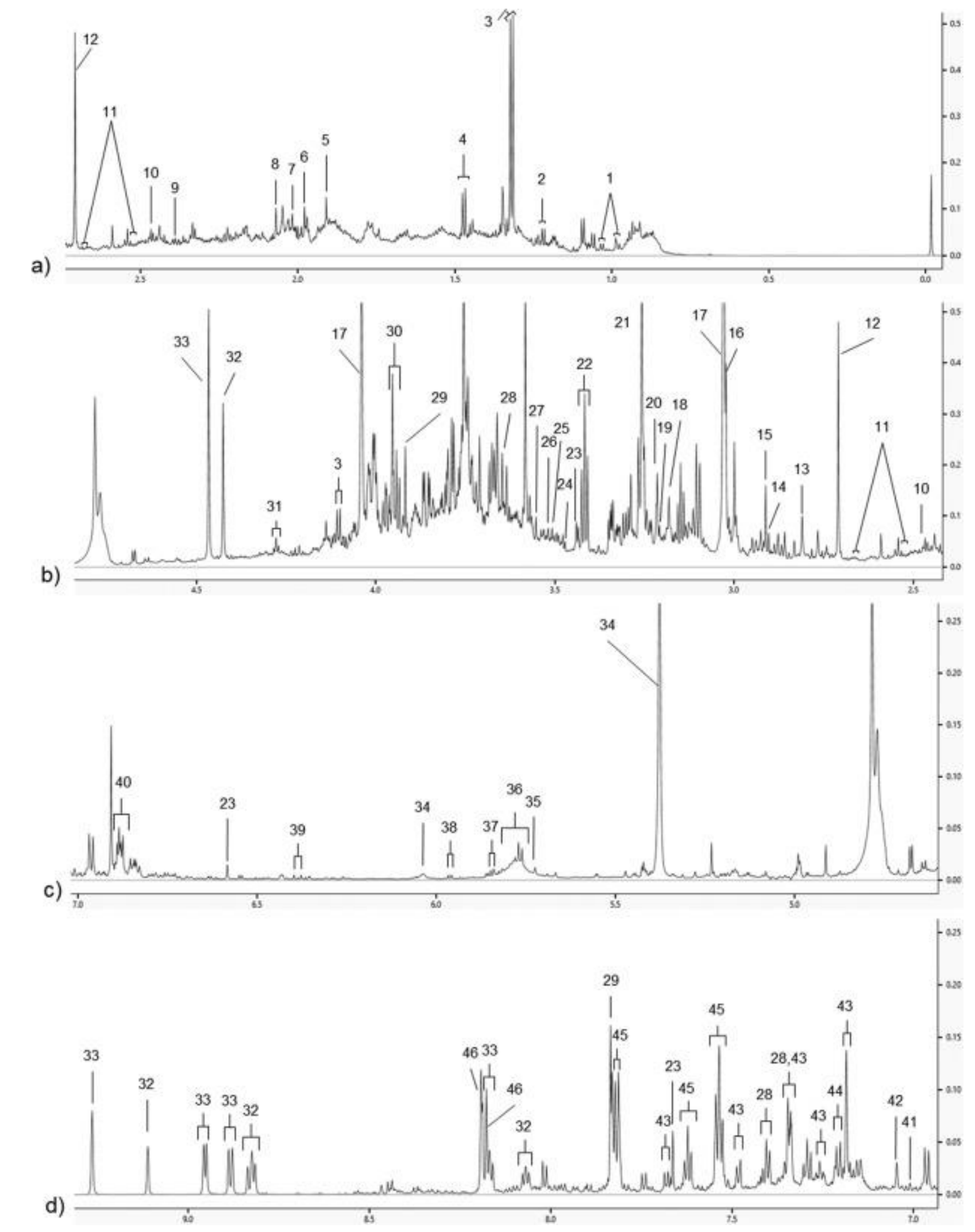

1. Download: Download high-res image (265KB) 


\section{Download: Download full-size image}

Fig. 3. Representative spectrum of urine from an healthy dog. For a better visualisation, the spectrum has been divided into four parts. a) From 0.0 to $2.8 \mathrm{ppm}$; b) from 2.4 to $4.9 \mathrm{ppm}$; c) from 4.6 to $7.0 \mathrm{ppm}$; d) from 7.0 to $10.0 \mathrm{ppm}$. The reported metabolites are: 1 valine; 2 fucose; 3 lactate; 4 alanine; 5 acetate; $6 \mathrm{N6}$-acetyllysine; $7 \mathrm{~N}$-acetylgycine; 8 acetylcisteine; 9 succinate; 10 pyridoxamine; 11 citrate; 12 dimethylamine; 13 methylguanidine; 14 trimethylamine; $15 \mathrm{~N}, \mathrm{~N}$-dimethylglycine; 16 creatine; 17 creatinine; 18 choline; 19 phosphorylcholine; 20 carnitine; 21 taurine, trimethylamine $\mathrm{N}$-oxyde, betaine; 22 taurine; 23 trans-aconitate; 24 3-hydroxyphenilacetate; 25 3-methylxantine; 26 2-hydroxyphenilacetate; 27 glycine; $28 \mathrm{~N}$-phenyilacetylglicine; 29 7-methylxantine; 30 creatine, creatine phosphate, glycolate; 31 pseudouridine; 32 trigonelline; 331 methylnicotinamide; 34 allantoine; 35 cis-aconitate; 36 urea; 37 xanthosine; 38 cytosine; 39 urocanate; 40 tyramine, tyrosine; 41 1-methylhistidine; 42 histidine; 43 3indoxylsulphate; 44 tyramine; 45 hippurate; 46 hypoxanthine.

Table 4. Assigned metabolites in the urine of healthy dogs.

\begin{tabular}{llcc}
\multicolumn{1}{c}{ Query } & HMDB & PubChem & KEGG \\
1,7-Dimethylxanthine & HMDB0001860 & 4687 & C13747 \\
1-Methyladenosine & HMDB0003331 & 27476 & C02494 \\
1-Methylguanine & HMDB0003282 & 70315 & C04152 \\
1-Methylhistidine & HMDB0000001 & 92105 & C01152 \\
1-Methylnicotinamide & HMDB0000699 & 457 & C02918 \\
2-Furoylglycine & HMDB0000439 & 21863 & NA \\
2-Hydroxybutyric acid & HMDB0000008 & 11266 & C05984 \\
2-Hydroxyphenylacetic acid & HMDB0000669 & 11970 & C05852 \\
2-Ketobutyric acid & HMDB0000005 & 58 & C00109 \\
2-Methylglutaric acid & HMDB0000422 & 12046 & NA \\
3-Hminoisobutyric acid & HMDB0003911 & 64956 & C05145 \\
3-Hydroxyphenylacetic acid & HMD0000440 & 12122 & \\
\hline
\end{tabular}




\section{Query}

3-Indoxylsulfic acid

3-Methyl-2-oxovaleric acid

3-Methylglutaric acid

3-Methylxanthine

4-Aminohippuric acid

4-Hydroxybenzoic acid

4-Hydroxyphenylacetic acid

4-Pyridoxic acid

7-Methyladenine

7-Methylxanthine

Acetic acid

Acetylcisteine

Adenosine

Alanine

Allantoin

Arabinitol

Ascorbic acid

Betaine

Carnitine

Choline

cis-Aconitic acid
HMDB

HMDB0000682

HMDB0000491

HMDB0000752

HMDB0001886

HMDB0001867

HMDB0000500

HMDB0000020

HMDB0000017

HMDB0011614

HMDB0001991

HMDB0000042

HMDB0001890

HMDB0000050

HMDB0000161

HMDB0000462

HMDB0001851

HMDB0000044

HMDB0000043

HMDB0000062

HMDB0000097

HMDB0000072
PubChem

10258

NA

47

C03465

12284

NA

70639

C16357

2148

NA

135

C00156

127

C00642

6723

C00847

71593

C02241

68374

C16353

176

C00033

12035

C06809

60961

C00212

5950

C00041

204

C01551

439255

C00532

54670067

C00072

247

C00719

2724480

C00318

305

C00114

643757

C00417 


\section{Query}

Citric acid

Creatine

Creatine phosphate

Creatinine

Cytosine

Dimethylamine

Ferulic acid

Formic acid

Fucose

Galactonic acid

Galactose

Glucaric acid

Glucuronic acid

Glycine

Glycolic acid

Glyoxylic acid

Hippuric acid

Histidine

Hypoxanthine

3-Methylhistidine

Indole-3-lactic acid
HMDB

HMDB0000094

HMDB0000064

HMDB0001511

HMDB0000562

HMDB0000630

HMDB0000087

HMDB0000954

HMDB0000142

HMDB0000174

HMDB0000565

HMDB0000143

HMDB0000663

HMDB0000127

HMDB0000123

HMDB0000115

HMDB0000119

HMDB0000714

HMDB0000177

HMDB0000157

HMDB0000479

HMDB0000671
311

C00158

586

C00300

587

C02305

588

C00791

597

C00380

674

C00543

445858

C01494

284

C00058

17106

C01019

128869

C00880

439357

C00984

33037

C00818

444791

C00191

750

C00037

757

C00160

760

C00048

464

C01586

6274

C00135

790

C00262

64969

C01152

92904

C02043 


\section{Query}

Isobutyric acid

Isoleucine

Kynurenic acid

Lactic acid

Lysine

Mannitol

Methylguanidine

$\mathrm{N}, \mathrm{N}$-Dimethylglycine

N6-Acetyllysine

$\mathrm{N}$-Acetylglycine

$\mathrm{N}$-Phenylacetylglycine

Oxoglutaric acid

Phosphorylcholine

Pseudouridine

Pyridoxamine

Serine

Succinic acid

Taurine

Threonine

trans-Aconitic acid

Trigonelline
HMDB

HMDB0001873

HMDB0000172

HMDB0000715

HMDB0000190

HMDB0000182

HMDB0000765

HMDB0001522

HMDB0000092

HMDB0000206

HMDB0000532

HMDB0000821

HMDB0000208

HMDB0001565

HMDB0000767

HMDB0001431

HMDB0000187

HMDB0000254

HMDB0000251

HMDB0000167

HMDB0000958

HMDB0000875
PubChem

6590

C02632

6306

C00407

3845

C01717

107689

C00186

5962

C00047

6251

C00392

10111

C02294

673

C01026

92832

C02727

10972

NA

68144

C05598

51

C00026

1014

C00588

15047

C02067

1052

C00534

5951

C00065

1110

C00042

1123

C00245

6288

C00188

444212

C02341

5570

C01004 


\begin{tabular}{|c|c|c|c|}
\hline Query & HMDB & PubChem & KEGG \\
\hline Trimethylamine & HMDB0000906 & 1146 & C00565 \\
\hline Trimethylamine $\mathrm{N}$-oxide & HMDB0000925 & 1145 & C01104 \\
\hline Tryptophan & HMDB0000929 & 6305 & $\mathrm{C} 00078$ \\
\hline Tyramine & HMDB0000306 & 5610 & $\mathrm{C} 00483$ \\
\hline Tyrosine & HMDB0000158 & 6057 & C00082 \\
\hline Uracil & HMDB0000300 & 1174 & C00106 \\
\hline Urea & HMDB0000294 & 1176 & C00086 \\
\hline Urocanic acid & HMDB0000301 & 736715 & C00785 \\
\hline Valine & HMDB0000883 & 6287 & C00183 \\
\hline Xanthine & HMDB0000292 & 1188 & C00385 \\
\hline Xanthosine & HMDB0000299 & 64959 & C01762 \\
\hline Xanthurenic acid & HMDB0000881 & 5699 & C02470 \\
\hline
\end{tabular}

Table 5. Significant pathways obtained by the pathway analysis module of MetaboAnalyst.

Pathway

Total $\stackrel{a}{ }$ Hits $\underline{b} \quad$ Raw $p$

Metabolites

Glycine, serine and threonine metabolism

$48 \quad 10 \quad 1.94 \mathrm{E}-06$

Phenylalanine

metabolism

Aminoacyl-tRNA

biosynthesis

Caffeine metabolism
$75 \quad 10$

0.00012

$45 \quad 8 \quad 7.59 \mathrm{E}-05$

$75-10-00012$

216
Serine; Choline; Betaine; Dimethylglycine; Glycine; Threonine; Creatine; 2-Ketobutyric acid; Glyoxylic acid; Tryptophan

Hippuric acid; N-Phenylacetylglycine; Succinic acid; 2Hydroxyphenylacetic acid; 4-Hydroxybenzoic acid; 4Hydroxyphenylacetic acid; Tyrosine; 3-Hydroxyphenylacetic acid

Histidine; Glycine; Serine; Valine; Alanine; Lysine; Isoleucine; Threonine; Tryptophan; Tyrosine

1,7-Dimethylxanthine; 3-Methylxanthine; 7-Methylxanthine; Xanthosine; Xanthine; Glyoxylic acid 


\begin{tabular}{|c|c|c|c|c|}
\hline Pathway & Total a & Hits $\underline{b}$ & Raw p & Metabolites \\
\hline Methane metabolism & 34 & 6 & 0.000666 & $\begin{array}{l}\text { Glycine; Formic acid; Trimethylamine; Trimethylamine N- } \\
\text { oxide; Dimethylamine; Serine; }\end{array}$ \\
\hline $\begin{array}{l}\text { Glyoxylate and } \\
\text { dicarboxylate } \\
\text { metabolism }\end{array}$ & 50 & 7 & 0.001004 & $\begin{array}{l}\text { cis-Aconitic acid; Glyoxylic acid; Oxoglutaric acid; Formic acid; } \\
\text { Glycolic acid; Citric acid; Succinic acid; }\end{array}$ \\
\hline Nitrogen metabolism & 39 & 6 & 0.001418 & Tyrosine; Tryptophan; Taurine; Histidine; Glycine; Formic acid; \\
\hline $\begin{array}{l}\text { Citrate cycle (TCA } \\
\text { cycle) }\end{array}$ & 20 & 4 & 0.00349 & Succinic acid; Oxoglutaric acid; cis-Aconitic acid; Citric acid; \\
\hline $\begin{array}{l}\text { Propanoate } \\
\text { metabolism }\end{array}$ & 35 & 5 & 0.005027 & $\begin{array}{l}\text { 2-Ketobutyric acid; Succinic acid; Lactic acid; 2-Hydroxybutyric } \\
\text { acid; Valine; }\end{array}$ \\
\hline $\begin{array}{l}\text { Valine, leucine and } \\
\text { isoleucine } \\
\text { biosynthesis }\end{array}$ & 27 & 4 & 0.010619 & Threonine; Valine; Isoleucine; 2-Ketobutyric acid; \\
\hline $\begin{array}{l}\text { Taurine and } \\
\text { hypotaurine } \\
\text { metabolism }\end{array}$ & 20 & 3 & 0.025942 & Taurine; Alanine; Acetic acid; \\
\hline Purine metabolism & 92 & 8 & 0.029144 & $\begin{array}{l}\text { Xanthine; Adenosine; Xanthosine; Hypoxanthine; Urea; } \\
\text { Glyoxylic acid; Glycine; Allantoin }\end{array}$ \\
\hline $\begin{array}{l}\text { Alanine, aspartate and } \\
\text { glutamate metabolism }\end{array}$ & 24 & 3 & 0.041947 & Alanine; Oxoglutaric acid; Succinic acid; \\
\hline $\begin{array}{l}\text { Pyrimidine } \\
\text { metabolism }\end{array}$ & 60 & 5 & 0.044772 & $\begin{array}{l}\text { Cytosine; Uracil; Pseudouridine; Urea; 3-Aminoisobutanoic } \\
\text { acid; }\end{array}$ \\
\hline
\end{tabular}

a

Total metabolites belonging to the pathway as reported by the pathway analysis module of MetaboAnalyst.

b

Metabolites assigned in urine of healthy dogs belonging to the pathway as obtained by the pathway analysis module of MetaboAnalyst. 
By univariate $t$-test, 83 buckets resulted significantly different between healthy and CKD dog urine samples. Unsupervised multivariate analysis (PCA) was able to distinguish between healthy and CKD dogs (Fig. 4). The supervised multivariate analysis using PLS-DA (Fig. 4, Table 6) indicated that the optimal model comprised 5 components $\left(\mathrm{R}^{2}=0.99, \mathrm{Q}^{2}=0.74\right)$, but also the model with only one component had reasonable predictive value $\left(\mathrm{R}^{2}=0.73, \mathrm{Q}^{2}=0.62\right)$. Both univariate and multivariate analysis were used to identify the differentially abundant metabolites. Of the 83 significantly different buckets, 21 were assigned to 17 metabolites (Table 6). The metabolites showing the highest increase in CKD samples were carnosine, 7methylxanthine and cis-aconitic acid, while the metabolites showing the most evident decrease were trigonelline and urocanic acid.
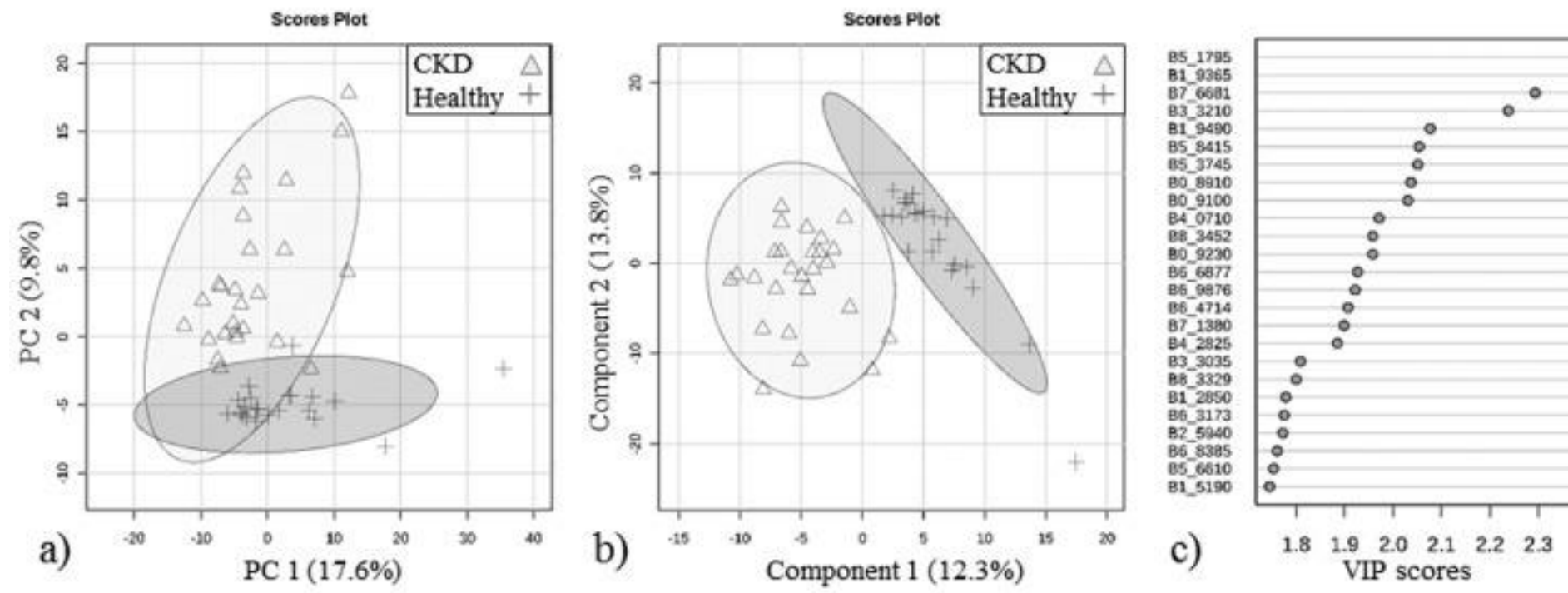

1. Download: Download high-res image (342KB)

2. Download : Download full-size image

Fig. 4. a) Principal component analysis (PCA) score plot of healthy (crosses and dark grey circle) and CKD (triangles and faint grey circle) urine samples. b) Partial Least

Square - Discriminant Analysis (PLS-DA) distribution plot of healthy (crosses and dark grey circle) and CKD (triangles and faint grey circle) urine samples. c) Variable Importance in Projection (VIP) scores for the 25 most influential buckets of PLS-DA. Table 6. Metabolites showing significant differences between healthy and CKD dogs.

Bucket

Metabolite
Fold change CKD/Healthy
VIP score
$P$ value
B6_9876 Carnosine
1.922 


\begin{tabular}{|c|c|c|c|c|}
\hline Bucket & Metabolite & Fold change CKD/Healthy & VIP score & $P$ value \\
\hline B3_9190 & 7-Methylxanthine & 2.94 & 1.444 & 0.037 \\
\hline B5_6610 & cis-Aconitic acid & 2.67 & 1.754 & 0.014 \\
\hline B2_7085 & Dimethylamine & 1.86 & 1.512 & 0.017 \\
\hline B2_8135 & Methylguanidine & 1.80 & 1.415 & 0.025 \\
\hline B7_8490 & Kynurenic acid & 1.77 & 1.333 & 0.045 \\
\hline B5_8415 & Xanthosine & 1.72 & 2.054 & 0.002 \\
\hline B4_2825 & Pseudouridine & 1.70 & 1.886 & 0.002 \\
\hline B7_6681 & Pseudouridine & 1.59 & 2.294 & 0.000 \\
\hline B5_3745 & Allantoin & 1.47 & 2.051 & 0.007 \\
\hline B7_3740 & Urocanic acid & 0.49 & 1.323 & 0.032 \\
\hline B0_9355 & 2-Hydroxybutyrric acid & 0.48 & 1.343 & 0.005 \\
\hline B1_0360 & Valine & 0.44 & 1.583 & 0.010 \\
\hline B7_7874 & 4-Hydroxybenzoic acid & 0.40 & 1.278 & 0.042 \\
\hline B7_1303 & Ferulic acid & 0.34 & 1.566 & 0.009 \\
\hline B8_1155 & 7-Methyladenine & 0.32 & 1.360 & 0.030 \\
\hline B7_7217 & Indole-3-lactic acid & 0.26 & 1.450 & 0.018 \\
\hline B6_3648 & Ferulic acid & 0.26 & 1.590 & 0.009 \\
\hline B6_3739 & Urocanic acid & 0.21 & 1.713 & 0.005 \\
\hline B8_8262 & Trigonelline & 0.15 & 1.284 & 0.043 \\
\hline B9_1121 & Trigonelline & 0.10 & 1.345 & 0.034 \\
\hline
\end{tabular}

a 
Variable Importance in Projection (VIP) scores.

\section{Discussion}

The aim of the present research was to characterise the urinary proteome and metabolome in healthy dogs and to compare it with that of urine collected from CKD patients, to suggest biomarkers of the disease that would be useful in veterinary medicine.

In the present study, SDS-PAGE allowed the separation of the urinary proteins based on their molecular mass, giving information about the localisation of the nephronal damage. Most urine samples (17/28) of CKD dogs analysed in this study had protein bands at both high and low MM, indicating a mixed glomerular and tubular pattern. It is generally recognised that the renal proteinuria with an UPC $>2$ is strongly indicative of glomerular involvement $[\underline{41}, \underline{42}]$. Our data support this evidence, as the electrophoretic profiles of the seven urine samples with an UPC $>2$ were characterised by protein bands with high MM. However, in all these samples, bands with low MM were also present, suggesting a concomitant tubular damage. Other authors reported a tubular impairment in dogs with UPC > 2 [42, $\underline{43}]$. On the other hand, in our study, 7 of the 21 samples with UPC $<2$ indicated also a glomerular involvement and hence the evaluation of proteinuria by UPC could lead to misinterpretation regarding the nephronal origin of the proteinuria, as previously suggested by other authors [41,[43], [44], [45], [46]].

In the present study, 8 dogs with early stages of CKD (I and II; serum creatinine < $2.1 \mathrm{mg} / \mathrm{dL}$ ), classified as non-proteinuric (UPC < 0.2 ) or borderline proteinuric (UPC $0.2-0.5)$, showed altered electrophoretic profiles with the decrease of uromodulin and the increase in number and intensity of low MM bands. Chacar et al., [43] also reported the prevalence of tubular pattern in urine samples of dogs with early stages of CKD. On the other hand, out of 14 dogs affected by CKD at advanced IRIS stages (III and IV; serum creatinine $>2.1 \mathrm{mg} / \mathrm{dL}$ ), 10 patients had a mixed profile, while 4 dogs presented a clear tubular pattern, with absent or mild glomerular involvement. Tubular epithelium seems to be more susceptible to ongoing stress and dysregulation promoting interstitial inflammation and fibrosis [47]. Therefore, it can be hypothesised that, in general, dogs with CKD in the initial phases (serum creatinine 
$<2.1 \mathrm{mg} / \mathrm{dL}$ and UPC < 0.5) might present a prevalent tubulointerstitial involvement followed by a gradual glomerular impairment leading to an increase of albumin and higher MM proteins in urine. In this complex scenario, the analysis of qualitative proteinuria could be essential to better characterise the kidney damage and the nephronal involvement.

In addition to the evaluation of the electrophoretic protein profiles, SDS-PAGE allowed the quantification of urinary uromodulin and albumin. In urine samples of healthy dogs, the abundance of uromodulin associated with the low concentration of albumin is confirmatory of data previously reported by other authors [43, [48], [49], [50]]. In our study, the quantification of these two proteins, followed by UMC and UAC calculation, represents an additional step for their clinical use. In fact, uromodulin and albumin are known markers of renal dysfunction or damage, in particular of CKD [ 51, 52]. Raila et al., [이 reported a decrease of uromodulin in azotaemic and proteinuric dogs affected by renal disease and, despite the different method used for protein quantification (western blot), UMC values determined in the healthy dogs were comparable to our results. In addition, Chacar et al., [43] quantified uromodulin by western blotting and reported a decrease of uromodulin only in the late stages of CKD (IRIS 3-4), suggesting this protein as a marker of CKD progression rather than of early diagnosis. Differently, in the present study, the decrease of uromodulin was observed by SDS-PAGE already in stage 1 non-proteinuric CKD dogs, suggesting uromodulin as a promising and early biomarker of renal dysfunction in dogs.

Urinary albumin concentration is low in healthy dogs and an increase occurs in the presence of renal involvement $[11, \underline{53}]$. Accordingly, in the present study, healthy dogs have low values of albumin and UAC, in the range of those reported by other authors [11, [54], [55], [56], [57]]. Different authors determined albuminuria in dogs affected by a variety of diseases and conditions, including CKD [11,[53], [54], [55], [56], [58], [59], [60], [61], [62]]. However, despite the clinical importance of albumin quantification in urine, the reference intervals for albuminuria are still lacking for dogs and should be the aim of further research. Finally, the presence of arginine esterase in urine of entire male dogs was also evidenced and needs to be considered to correctly interpret urine electrophoretic profile and to exclude false tubular involvement as previously reported [15,42]. 
The second part of the study focused on the application of NMR to characterise the urinary metabolome of healthy dogs and to evaluate the differences with CKD patients. As most CKD samples contained high protein concentrations, to avoid interferences on NMR spectra and possible false positives, a further step in sample preparation was added by precipitating the proteins. This step allowed the enrichment of urine metabolome, improving the quality of the spectra and the identification of a higher number of metabolites. Moreover, since the high repeatability of NMR metabolomics is well known [20,21], no technical replicates were analysed and only biological replicates were considered. From the corresponding spectra, 86 metabolites were identified in healthy samples, a number higher than those previously reported in dog urine by other authors $[\underline{32}, \underline{33}, \underline{63}, \underline{64]}$ and producing, so far and to the best of our knowledge, one of the most complete dataset of canine urinary metabolome. Most of these metabolites are of endogenous origin, while others, like ferulic acid, are of exogenous or mixed origin. Most of the identified metabolites were previously reported in urine of healthy or diseased dogs $[\underline{32}, \underline{33}, \underline{65}]$, in human urine $[\underline{19}, \underline{22}]$ and also in feline urine [31]. The majority of these metabolites is involved in amino acids metabolism, purine and pyrimidine metabolism, tricarboxylic acid cycle and methane metabolism.

Nine metabolites were significantly increased in urine of CKD dogs. Carnosine, a dipeptide composed by alanine and histidine acting as an antioxidant scavenger, showed the most evident increase. This molecule is filtered by the glomerulus and then reabsorbed at the level of the proximal tubule by the proton-coupled oligotransporter PEPT2 [66]. It has been recently reported that the kidney has an intrinsic carnosine metabolism with carnosine synthase and carnosinase 1 activity in the glomeruli and tubular cells $[67,68]$. In CKD dogs, the increased urinary excretion of carnosine may reflect an oxidative stress suffered by the kidney, a condition hypothesised also by other authors in obese dogs $[\underline{32}, \underline{65]}$. Moreover, since carnosine is present at high concentrations in muscle tissues, and muscle weakness and atrophy are common findings in CKD patients, the increase of this molecule in urine may also reflect an increased muscle catabolism [65]. Finally, as a causative event, a damage of the epithelium of the proximal tubule might also be hypothesised, leading to impaired reabsorption of carnosine; this hypothesis is supported by the decrease of uromodulin evidenced by SDS-PAGE and by the increase of cis-aconitic acid in urine of CKD 
dogs. Cis-aconitic acid, an intermediate in the tricarboxylic acid cycle, was observed in the urine of type 2 diabetic human patients. In fact, increased excretion of this metabolite reflects local effects on tubular transport in the kidneys [69]. Therefore, a damage of the tubular epithelium might determine an inefficient reabsorption leading to an increased concentration of urinary cis-aconitic acid and carnosine.

Regarding other urinary metabolites increased in urine of CKD dogs, xanthosine, allantoin, and 7-methylxanthine are of interest. These metabolites belong to the complex pathways of purine metabolism; in particular, during purine catabolism, the nucleoside xanthosine is transformed into xanthine, which in turn is oxidized to uric acid by uricase. In humans, uric acid is the end product of purine catabolism, while in dogs an additional reaction transforms this metabolite into allantoin. In humans, some of these metabolites were suggested as possible markers of diabetic nephropathy [미], end stage renal disease [71] or other kidney disorders [72], while an increase of allantoin and xanthine to creatinine ratios were previously reported in urine of dogs affected by CKD [73]. Despite the possible influence of medications, such as allopurinol or diuretics received by two CKD dogs included in the present study, that could have affected purine metabolism, these data show evidence that CKD is associated with alterations in urinary concentrations of purine metabolites, and thus, this issue deserves more attention in further research.

Three additional metabolites increased in urine of CKD dogs. They were methylguanidine (MG), kynurenic acid (KnA) and dimethylamine (DA). These molecules are well known uremic toxins that accumulate in serum and urine due to the impairment of renal function $[\underline{74}, \underline{75]}$. MG derives from creatinine and is often detected in serum and urine of uremic human patients [76,77]. MG was detected also in serum of uremic dogs and was shown to increase in urine of dogs affected by transitional cell carcinoma [33,78]. In the present study, 14 samples were collected from dogs at advanced CKD stages (serum creatinine > $2.1 \mathrm{mg} / \mathrm{dL}$; IRIS 3 and 4). Therefore, the increase of MG in urine of CKD dogs might be considered in further studies as a possible biomarker of advanced CKD stages. KnA is a key inflammatory metabolite of the tryptophan catabolic pathway: the degradation of tryptophan occurs through the formation of kynurenine, which in turn can be transformed into KnA and other related metabolites. Kidneys are involved in tryptophan metabolism either eliminating the catabolites or producing the enzymes involved in tryptophan 
metabolism. In case of renal failure, these metabolites, which are physiologically excreted in urine, accumulate in the blood, contributing to uremia. Accordingly, the study of Rhee et al., [79] reported that serum levels of KnA increased with CKD development and severity. Moreover, increased KnA urinary excretion was associated with adverse clinical outcomes in critically ill patients with acute kidney injury [80] and four tryptophan metabolites, including urinary $\mathrm{KnA}$, were reported to be associated with an estimated glomerular filtration rate (eGFR) decline and with oxidative stress after eight years follow-up $[\underline{81}, \underline{82}]$.

Eight metabolites were significantly reduced in urine of CKD patients and the most consistent decrease was evident for trigonelline, which can be obtained from the diet, or alternatively produced as a niacin-derived metabolite. Proximal tubule epithelia synthesize NAD from precursors taken up from urine and an excess of metabolites of the biosynthetic pathway, including trigonelline, is normally secreted in urine. In case of tubular damage, a reduced/absent absorption of nicotinamide or nicotinic acid occurs leading to a reduced/absent trigonelline secretion. Accordingly, in a mice model of acute kidney injury, trigonelline removal from urine was reported as a consequence of tubular damage [[83], [84], [85]].

Significant decrease was observed also for urocanic, indole-3-lactic and ferulic acids. The two first metabolites derive from hepatic histidine and tryptophan catabolism, respectively. In particular, histidine can be converted to histamine, 3-methylhistidine or urocanic acid by different pathways, while indole-3-lactic acid is obtained through the reduction of indolepyruvic acid derived by oxidative deamination of tryptophan. Finally, ferulic acid is a phenolic acid widely distributed in plants that can be absorbed by the small intestine and excreted through the urine. All these metabolites can be found in plasma and urine $[22, \underline{31}, \underline{86}$. Serum indole-3-lactic acid was recently associated to eGFR in human CKD patients [87], but, to the best of our knowledge, no information is available in the literature on the decrease of these metabolites in the urine of CKD patients. Further studies are therefore needed to clarify their role as possible biomarkers.

This study presents some limitations. The first one is related to the different age between healthy and diseased dogs. Since CKD is a disease of older animals, and adult/old dogs are usually presented to the Veterinary Teaching Hospital due to pathologic conditions, it was not possible to collect samples from age-matched 
controls. However, none of the different metabolites identified between healthy and CKD dogs were reported by Wang et al., [88] as affected by age in healthy dogs. Therefore, despite a possible age effect on urine metabolome cannot be completely excluded, we hypothesise that the effect of CKD was more consistent than the effect of the age. In addition, numerous dog breeds were included in the two study groups; therefore, breed-specific differences or effects on the urinary proteome and metabolome could not be determined in the present study. Secondly, the limited number of CKD samples did not allow to highlight significant differences among CKD stages for both proteomics and metabolomics results and it was not possible to highlight clear trends in biomarkers as the disease worsens. The final limitation relates to the absence of technical replicates for the evaluation of the robustness of our data. The technical evaluation of the performance of NMR applied to the dog urine was out of the scope of the present research, especially since the high repeatability of NMR metabolomics is well-known [20,21].

\section{Conclusions}

The integrated application of proteomics and metabolomics on urine samples yielded new insight into the molecular complexity of urine in healthy dogs and highlighted biochemical changes in response to CKD. SDS-PAGE evidenced the involvement of the tubular compartment with the decrease of uromodulin and the presence of low MM bands also in non-proteinuric and non-azotaemic dogs and could be considered a useful and complementary diagnostic tool for clinical pathologists, clinicians and researchers working in veterinary nephrology and urology.

NMR metabolomics was successfully applied to canine urinary samples allowing the identification of 86 metabolites. Of these, 17 showed significant differences in CKD dogs. In particular, the increase of carnosine and cis-aconitic acid and the decrease of trigonelline are indicative of the tubular involvement, adding further evidence to the results of SDS-PAGE. Additional studies are needed to clarify the molecular mechanisms underlying the pathophysiology of CKD and to confirm the role of the discovered metabolites as biomarkers of this disease in dogs. In particular, increasing the number of urine samples collected from dogs affected by all stages of CKD should 
be the focus of future research to confirm early biomarkers and highlight trends as the disease worsens.

\section{Funding}

EF was supported by a study grant from the Fondazione ItPA Onlus (2017, Rome, Italy). GI was supported by a grant from University of Bologna (RFO). The NMR data were acquired at CERMAX, ITQB-NOVA, Oeiras, Portugal with equipment funded by FCT, project AAC 01/SAICT/2016 and ONEIDA (LISBOA-01-0145FEDER-016417) which is co-funded by FEEI - "Fundos Europeus Estruturais e de Investimento" from "Programa Operacional Regional Lisboa 2020" and by national funds from FCT.

The funders had no role in study design, data collection and analysis, decision to publish, or preparation of the manuscript.

\section{Author contribution}

EF processed and analysed samples for proteomics and metabolomics, analysed and interpreted data and wrote the manuscript. GI designed and supervised the study, interpreted data, wrote and critically revised the manuscript. FD supervised the recruitment of the patients, interpreted data and critically revised the manuscript. GA interpreted data and critically revised the manuscript. KV recruited the patients, collected samples, performed urine, blood and serum biochemistry and critically revised the manuscript. EB performed protein mass identification and critically revised the manuscript. AMA designed and supervised the study and critically revised the manuscript. MM acquired and analysed metabolomics data and critically revised the manuscript. All authors read and approved the final manuscript.

\section{Declaration of Competing Interest}

None

\section{Appendix A. Supplementary data}

Download : Download spreadsheet (20KB) 
Supplementary Table 1. Clinical data, serum and urine biochemistry of each dog included in the study.

\section{References}

[1]

D.J. Polzin

Chronic kidney disease in small animals

Vet. Clin. North Am. Small Anim. Pract., 41 (2011), pp. 15-30, 10.1016/i.cvsm.2010.09.004

ArticleDownload PDFView Record in ScopusGoogle Scholar

[2]

D.G. O’Neill, J. Elliott, D.B. Church, P.D. Mcgreevy, P.C. Thomson, D.C. Brodbelt

Chronic kidney disease in dogs in UK veterinary practices: prevalence, risk factors, and survival J. Vet. Intern. Med., 27 (2013), pp. 814-821, 10.1111/jvim.12090

CrossRefView Record in ScopusGoogle Scholar

$[3]$

J.W. Bartges

Chronic kidney disease in dogs and cats

Vet. Clin. North Am. Small Anim. Pract., 42 (2012), pp. 669-692, 10.1016/j.cvsm.2012.04.008

ArticleDownload PDFView Record in ScopusGoogle Scholar

[4]

International Renal Interest Society

IRIS Staging of CKD (modified 2019)

(2019), pp. 1-8

View Record in Scopus Google Scholar

[5]

S. Kovarikova

Indirect markers of glomerular filtration rate in dogs and cats: A review

Vet. Med. (Praha), 63 (2018), pp. 395-412, 10.17221/77/2017-VETMED

CrossRefView Record in ScopusGoogle Scholar

[6] 
P. Pocar, P. Scarpa, A. Berrini, P. Cagnardi, R. Rizzi, V. Borromeo

Diagnostic potential of simplified methods for measuring glomerular filtration rate to detect chronic kidney disease in dogs

J. Vet. Intern. Med., 33 (2019), pp. 2105-2116, 10.1111/jvim.15573

CrossRefView Record in ScopusGoogle Scholar

J.A. Hokamp, M.B. Nabity

Renal biomarkers in domestic species

Vet. Clin. Pathol., 45 (2016), pp. 28-56, 10.1111/vcp.12333

CrossRefView Record in ScopusGoogle Scholar

[8]

E. Ferlizza, A. Campos, A. Neagu, A. Cuoghi, E. Bellei, E. Monari, F. Dondi, A.M. Almeida, G. Isani

The effect of chronic kidney disease on the urine proteome in the domestic cat (Felis catus)

Vet. J., 204 (2015), pp. 73-81, 10.1016/j.tvjl.2015.01.023

ArticleDownload PDFView Record in ScopusGoogle Scholar

[9]

E. Ferlizza, F. Dondi, G. Andreani, D. Bucci, J. Archer, G. Isani

Validation of an electrophoretic method to detect albuminuria in cats

J. Feline Med. Surg., 19 (2017), pp. 860-868, 10.1177/1098612X16664112

View Record in ScopusGoogle Scholar

[10]

I. van Hoek, E. Meyer, L. Duchateau, K. Peremans, P. Smets, S. Daminet

Retinol-binding protein in serum and urine of hyperthyroid cats before and after treatment with radioiodine

J. Vet. Intern. Med., 23 (2009), pp. 1031-1037, 10.1111/j.1939-1676.2009.0364.x

CrossRefView Record in ScopusGoogle Scholar

P.M.Y. Smets, E. Meyer, B.E.J. Maddens, L. Duchateau, S. Daminet

Urinary markers in healthy young and aged dogs and dogs with chronic kidney disease

J. Vet. Intern. Med., 24 (2010), pp. 65-72, 10.1111/i.1939-1676.2009.0426.x 
M.B. Nabity, G.E. Lees, L.J. Dangott, R. Cianciolo, J.S. Suchodolski, J.M. Steiner

Proteomic analysis of urine from male dogs during early stages of tubulointerstitial injury in a canine model of progressive glomerular disease

Vet. Clin. Pathol., 40 (2011), pp. 222-236, 10.1111/j.1939-165X.2011.00307.x

View Record in ScopusGoogle Scholar

T.L. Williams, J. Archer

Evaluation of urinary biomarkers for azotemic chronic kidney disease in cats

J. Small Anim. Pract., 57 (2015), pp. 122-129, 10.1111/jsap.12439

View Record in ScopusGoogle Scholar

G. Isani, E. Ferlizza, J.E. Nally

\section{Proteomic research in urine and other fluids}

A.M. de Almeida, P.D. Eckersall, I. Miller (Eds.), Proteomics Domestic Animals: From Farm to System Biology, Springer, Cham (2018), pp. 121-147, 10.1007/978-3-319-69682-9 7

CrossRefView Record in ScopusGoogle Scholar

I. Miller, A. PreßImayer-Hartler, R. Wait, K. Hummel, C. Sensi, I. Eberini, E. Razzazi-

Fazeli, E. Gianazza

In between - proteomics of dog biological fluids

J. Proteome, 106 (2014), pp. 30-45, 10.1016/j.jprot.2014.04.016

ArticleDownload PDFView Record in ScopusGoogle Scholar 
J. Kuleš, P. Bilić, B. Beer Ljubić, J. Gotić, M. Crnogaj, M. Brkljačić, V. Mrljak

Glomerular and tubular kidney damage markers in canine babesiosis caused by Babesia canis Ticks Tick. Borne. Dis., 9 (2018), pp. 1508-1517, 10.1016/j.ttbdis.2018.07.012

ArticleDownload PDFView Record in ScopusGoogle Scholar

L. Pelander, V. Brunchault, B. Buffin-

Meyer, J. Klein, B. Breuil, P. Zürbig, P. Magalhães, W. Mullen, J. Elliott, H. Syme, J.P. Schanstra, J. Häggström, I. Ljungvall

Urinary peptidome analyses for the diagnosis of chronic kidney disease in dogs

Vet. J., 249 (2019), pp. 73-79, 10.1016/j.tvjl.2019.05.010

ArticleDownload PDFView Record in ScopusGoogle Scholar

I.F. Duarte, S.O. Diaz, A.M. Gil

NMR metabolomics of human blood and urine in disease research

J. Pharm. Biomed. Anal., 93 (2014), pp. 17-26, 10.1016/j.jpba.2013.09.025

ArticleDownload PDFView Record in ScopusGoogle Scholar

R.A. Silva, T.C.S. Pereira, A.R. Souza, P.R. Ribeiro

1H NMR-based metabolite profiling for biomarker identification

Clin. Chim. Acta, 502 (2020), pp. 269-279, 10.1016/j.cca.2019.11.015

ArticleDownload PDFView Record in ScopusGoogle Scholar 
J.L. Markley, R. Brüschweiler, A.S. Edison, H.R. Eghbalnia, R. Powers, D. Raftery, D.S. Wishart

The future of NMR-based metabolomics

Curr. Opin. Biotechnol., 43 (2017), pp. 34-40, 10.1016/j.copbio.2016.08.001

$\underline{\text { ArticleDownload PDFView Record in ScopusGoogle Scholar }}$

P.G. Takis, V. Ghini, L. Tenori, P. Turano, C. Luchinat

Uniqueness of the NMR approach to metabolomics

Trends Anal. Chem., 120 (2019), p. 115300, 10.1016/i.trac.2018.10.036

ArticleDownload PDFView Record in ScopusGoogle Scholar

S. Bouatra, F. Aziat, R. Mandal, A.C. Guo, M.R. Wilson, C. Knox, T.C. Bjorndahl, R. Krishnamurthy, F. Saleem, P. Liu, Z.T. Dame, J. Poelzer, J. Huynh, F.S. Yallou, N. Psychogios, E. Dong, R. Bogumil, C. Roehring, D.S. Wishart

The human urine metabolome

PLoS One, 8 (2013), Article e73076, 10.1371/journal.pone.0073076

CrossRefView Record in ScopusGoogle Scholar

A. Julià, M. Vinaixa, E. Domènech, A. Fernández-

Nebro, J.D. Cañete, C. Ferrándiz, J. Tornero, J.P. Gisbert, P. Nos, A.G. Casbas, L. Puig, I. GonzálezÁlvaro, J.A. Pinto-

Tasende, R. Blanco, M.A. Rodríguez, A. Beltran, X. Correig, S. Marsal, E. Fernández, R. Sanmartí, J . Gratacós, V.M. Martínez-

Taboada, F. Gomollón, E. Daudén, J. Maymó, R. Queiró, F.J.L. Longo, E. Garcia-

Planella, J.L.S. Carazo, M. Alperi-López, C. Montilla, J.J. Pérez-Venegas, B. FernándezGutiérrez, J.L. Mendoza, J.L. López Estebaranz, À. Olivé, J.C. Torre-Alonso, M. Barreirode Acosta, D.M. Ramírez, H. Corominas, S. Muñoz-Fernández, J.L. Andreu, F. Muñoz, P. de la Cueva, A. Erra, C.M. González, M.Á. Aguirre-

Zamorano, M. Vera, F. Vanaclocha, D. Roig, P. Vela, C. Saro, E. Herrera, P. Zarco, J.M. Nolla, M. E 
steve, J.L. Marenco de la Fuente, J.M. Pego-Reigosa, V. García-

Sánchez, J. Panés, E. Fonseca, F. Blanco, J. Rodríguez-

Moreno, P. Carreira, J. Ramírez, G. Avila, L. Codó, J.L. Gelpí, A.C. García-

Montero, N. Palau, M. López-Lasanta, R. Tortosa, A. Alonso

Urine metabolome profiling of immune-mediated inflammatory diseases

BMC Med., 14 (2016), p. 133, 10.1186/s12916-016-0681-8

\section{$\underline{\text { View Record in Scopus Google Scholar }}$}

E.A. Mathé, A.D. Patterson, M. Haznadar, S.K. Manna, K.W. Krausz, E.D. Bowman, P.G. Shields, J.

R. Idle, P.B. Smith, K. Anami, D.G. Kazandjian, E. Hatzakis, F.J. Gonzalez, C.C. Harris

Non-invasive urinary metabolomic profiling identifies diagnostic and prognostic markers in lung cancer

Cancer Res., 74 (2014), pp. 3259-3270, 10.1158/0008-5472.CAN-14-0109

\section{View Record in ScopusGoogle Scholar}

C.L. Silva, A. Olival, R. Perestrelo, P. Silva, H. Tomás, J.S. Câmara

Untargeted urinary1H NMR-based metabolomic pattern as a potential platform in breast cancer detection

Metabolites., 9 (2019), p. 269, 10.3390/metabo9110269

CrossRefView Record in ScopusGoogle Scholar

E.R. Kim, H.N. Kwon, H. Nam, J.J. Kim, S. Park, Y.H. Kim

Urine-NMR metabolomics for screening of advanced colorectal adenoma and early stage colorectal cancer

Sci. Rep., 9 (2019), p. 4786, 10.1038/s41598-019-41216-y

View Record in ScopusGoogle Scholar 
M. Martin-Lorenzo, L. Gonzalez-Calero, A. Ramos-Barron, M.D. Sanchez-Niño, C. GomezAlamillo, J.M. García-Segura, A. Ortiz, M. Arias, F. Vivanco, G. Alvarez-Llamas

Urine metabolomics insight into acute kidney injury point to oxidative stress disruptions in energy generation and $\mathrm{H}_{2} \mathrm{~S}$ availability

J. Mol. Med., 95 (2017), pp. 1399-1409, 10.1007/s00109-017-1594-5

\section{CrossRefView Record in ScopusGoogle Scholar}

M. Posada-Ayala, I. Zubiri, M. Martin-Lorenzo, A. Sanz-Maroto, D. Molero, L. GonzalezCalero, B. Fernandez-Fernandez, F. De La

Cuesta, C.M. Laborde, M.G. Barderas, A. Ortiz, F. Vivanco, G. Alvarez-Llamas

Identification of a urine metabolomic signature in patients with advanced-stage chronic kidney disease

Kidney Int., 85 (2014), pp. 103-111, 10.1038/ki.2013.328

$\underline{\text { ArticleDownload PDFCrossRefView Record in ScopusGoogle Scholar }}$

M. Breit, K.M. Weinberger

Metabolic biomarkers for chronic kidney disease

Arch. Biochem. Biophys., 589 (2016), pp. 62-80, 10.1016/j.abb.2015.07.018

ArticleDownload PDFView Record in ScopusGoogle Scholar

S. Hallan, M. Afkarian, L.R. Zelnick, B. Kestenbaum, S. Sharma, R. Saito, M. Darshi, G. Barding, D. Raftery, W. Ju, M. Kretzler, K. Sharma, I.H. de Boer 
Metabolomics and gene expression analysis reveal down-regulation of the citric acid (TCA) Cycle in non-diabetic CKD patients

EBioMedicine., 26 (2017), pp. 68-77, 10.1016/j.ebiom.2017.10.027

ArticleDownload PDFView Record in ScopusGoogle Scholar

S.-M. Rivera-Vélez, N.F. Villarino

Feline urine metabolomic signature: characterization of low-molecular-weight substances in urine from domestic cats

J. Feline Med. Surg., 20 (2017), pp. 155-163, 10.1177/1098612X17701010

$\underline{\text { Google Scholar }}$

J. Soder, R. Hagman, J. Dicksved, S. Lindase, K. Malmlof, P. Agback, A. Moazzami, K. Hoglund, S. Wernersson

The urine metabolome differs between lean and overweight Labrador Retriever dogs during a feed-challenge

PLoS One, 12 (2017), Article e0180086, 10.1371/journal.pone.0180086

CrossRefView Record in ScopusGoogle Scholar

J. Zhang, S. Wei, L. Liu, G.A. Nagana Gowda, P. Bonney, J. Stewart, D.W. Knapp, D. Raftery

NMR-based metabolomics study of canine bladder cancer

Biochim. Biophys. Acta, 1822 (2012), pp. 1807-1814, 10.1016/j.bbadis.2012.08.001

ArticleDownload PDFView Record in ScopusGoogle Scholar 
Y.A. Lawrence, B.C. Guard, J.M. Steiner, J.S. Suchodolski, J.A. Lidbury

Untargeted metabolomic profiling of urine from healthy dogs and dogs with chronic hepatic disease

PLoS One, 14 (2019), Article e0217797, 10.1371/journal.pone.0217797

CrossRefView Record in ScopusGoogle Scholar

C. Zhu, V. Faillace, F. Laus, M. Bazzano, L. Laghi

Characterization of trotter horses urine metabolome by means of proton nuclear magnetic resonance spectroscopy

Metabolomics., 14 (2018), p. 106, 10.1007/s11306-018-1403-3

View Record in ScopusGoogle Scholar

G. Isani, E. Ferlizza, A. Cuoghi, E. Bellei, E. Monari, B. Bianchin Butina, C. Castagnetti

Identification of the most abundant proteins in equine amniotic fluid by a proteomic approach Anim. Reprod. Sci., 174 (2016), pp. 150-160, 10.1016/j.anireprosci.2016.10.003

ArticleDownload PDFView Record in ScopusGoogle Scholar

D. Jacob, C. Deborde, M. Lefebvre, M. Maucourt, A. Moing

NMRProcFlow: a graphical and interactive tool dedicated to 1D spectra processing for NMRbased metabolomics

Metabolomics., 13 (2017), p. 36, 10.1007/s11306-017-1178-y

View Record in ScopusGoogle Scholar 
J. Xia, D.S. Wishart

Using MetaboAnalyst 3.0 for comprehensive metabolomics data analysis

Curr. Protoc. Bioinforma, 55 (2016), 10.1002/cpbi.11

$\underline{\text { Google Scholar }}$

M. Palma, L.E. Hernández-Castellano, N. Castro, A. Arguëllo, J. Capote, M. Matzapetakis, A.M. de Almeida

NMR-metabolomics profiling of mammary gland secretory tissue and milk serum in two goat breeds with different levels of tolerance to seasonal weight loss

Mol. BioSyst., 12 (2016), pp. 2094-2107, 10.1039/C5MB00851D

View Record in ScopusGoogle Scholar

E. Szymańska, E. Saccenti, A.K. Smilde, J.A. Westerhuis

Double-check: validation of diagnostic statistics for PLS-DA models in metabolomics studies Metabolomics., 8 (2012), pp. 3-16, 10.1007/s11306-011-0330-3

CrossRefView Record in ScopusGoogle Scholar

J.A. Hokamp, R.E. Cianciolo, M. Boggess, G.E. Lees, S.L. Benali, M. Kovarsky, M.B. Nabity

Correlation of urine and serum biomarkers with renal damage and survival in dogs with naturally occurring proteinuric chronic kidney disease

J. Vet. Intern. Med., 30 (2016), pp. 591-601, 10.1111/jvim.13832

CrossRefView Record in ScopusGoogle Scholar 
J.A. Hokamp, S.A. Leidy, I. Gaynanova, R.E. Cianciolo, M.B. Nabity

Correlation of electrophoretic urine protein banding patterns with severity of renal damage in dogs with proteinuric chronic kidney disease

Vet. Clin. Pathol., 47 (2018), pp. 425-434, 10.1111/vcp.12648

CrossRefView Record in ScopusGoogle Scholar

F. Chacar, M. Kogika, T.R. Sanches, D. Caragelasco, C. Martorelli, C. Rodrigues, J.M.C. Capcha, D. Chew, L. Andrade

Urinary Tamm-Horsfall protein, albumin, vitamin D-binding protein, and retinol-binding protein as early biomarkers of chronic kidney disease in dogs

Physiol. Rep., 5 (2017), p. e13262, 10.14814/phy2.13262

CrossRefView Record in ScopusGoogle Scholar

R. Cianciolo, J. Hokamp, M. Nabity

Advances in the evaluation of canine renal disease

Vet. J., 215 (2016), pp. 21-29, 10.1016/j.tvjl.2016.04.012

ArticleDownload PDFView Record in ScopusGoogle Scholar

O. Jaturakan, M. Vanichwatanaramlouk, A. Kornkaew, M. Trisiriroj

SDS-PAGE electrophoresis for urinary protein analysis in dogs with chronic kidney disease and urinary tract infection

Thai J. Vet. Med., 43 (2013), pp. 75-83

View Record in ScopusGoogle Scholar 
D.S. Caragelasco, M.M. Kogika, C.R. Martorelli, K.K. Kanayama, D.M.N. Simões

Urine protein electrophoresis study in dogs with pituitary dependent hyperadrenocorticism during therapy with trilostane

Pesqui. Vet. Bras., 37 (2017), pp. 734-740, 10.1590/s0100-736×2017000700014

CrossRefView Record in ScopusGoogle Scholar

G. Segev, M.B. Nabity, G.F. Grauer, S. Brown, D.J. Polzin, L.D. Cowgill, C. Langston, A.M. van Dongen, J. Elliott

Is progressive chronic kidney disease a slow acute kidney injury?

Vet. Clin. North Am. Small Anim. Pract., 46 (2016), pp. 995-1013, 10.1016/j.cvsm.2016.06.001

$\underline{\text { Google Scholar }}$

J. Raila, H. Aupperle, G. Raila

Renal pathology and urinary protein excretion in a 14-month-old Bernese mountain dog with chronic renal failure

J. Vet. Med A., 54 (2007), pp. 131-135, 10.1111/i.1439-0442.2007.00919.x

CrossRefView Record in ScopusGoogle Scholar

J. Raila, L. Brunnberg

Influence of kidney function on urinary excretion of albumin and retinol-binding protein in dogs with naturally occurring renal disease

Am. J. Vet. Res., 71 (2010), pp. 1387-1394, 10.2460/ajvr.71.11.1387

CrossRefView Record in ScopusGoogle Scholar

J. Raila, F.J. Schweigert, B. Kohn

Relationship between urinary Tamm-Horsfall protein excretion and renal function in dogs with naturally occurring renal disease

Vet. Clin. Pathol., 43 (2014), pp. 261-265, 10.1111/vcp.12143

CrossRefView Record in ScopusGoogle Scholar 
O. Devuyst, E. Olinger, L. Rampoldi

Uromodulin: from physiology to rare and complex kidney disorders

Nat. Rev. Nephrol., 13 (2017), pp. 525-544, 10.1038/nrneph.2017.101

\section{CrossRefView Record in ScopusGoogle Scholar}

A. Okubo, A. Nakashima, S. Doi, T. Doi, T. Ueno, K. Maeda, R. Tamura, K. Yamane, T. Masaki

High-normal albuminuria is strongly associated with incident chronic kidney disease in a nondiabetic population with normal range of albuminuria and normal kidney function Clin. Exp. Nephrol. (2020), 10.1007/s10157-019-01842-2

\section{$\underline{\text { Google Scholar }}$}

J. Martinez, C. Kellogg, M.C. Iazbik, C.G. Couto, B.M. Pressler, T.M. Hoepf, M.J. Radin

The renin-angiotensin-aldosterone system in Greyhounds and non-Greyhound dogs

J. Vet. Intern. Med., 31 (2017), pp. 988-993, 10.1111/jvim.14720

\section{CrossRefView Record in ScopusGoogle Scholar}

A. Bacic, M.M. Kogika, K.C. Barbaro, C.S. Iuamoto, D.M.N. Simões, M.L. Santoro

Evaluation of albuminuria and its relationship with blood pressure in dogs with chronic kidney disease

Vet. Clin. Pathol., 39 (2010), pp. 203-209, 10.1111/j.1939-165X.2009.00207.x

View Record in ScopusGoogle Scholar

C.Z. Cavalcante, M.M. Kogika, A. Bacic, M.L. Santoro, S.I. Miyashiro, J.P. Sault, M.K. Oyafuso, D.M . Simões

Evaluation of albminuria and electrophoresis of urinary proteins from dogs with hyperadrenocorticism and relation with systemic arterial pressure

Pesqui. Vet. Bras., 33 (2013), pp. 1354-1363, 10.1590/S0100-736X2013001100013

$\underline{\text { Google Scholar }}$

P.M.Y. Smets, H.P. Lefebvre, B.P. Meij, S. Croubels, E. Meyer, I. Van de Maele, S. Daminet 
Long-term follow-up of renal function in dogs after treatment for ACTH-dependent hyperadrenocorticism

J. Vet. Intern. Med., 26 (2012), pp. 565-574, 10.1111/j.1939-1676.2012.00915.x

\section{CrossRefView Record in ScopusGoogle Scholar}

O.L. Ioannis, S. Nectarios, C. Jose Joaquin, T. Konstantina, R. Timoleon, P. Zoe

The prognostic value of microalbuminuria in puppies with canine parvoviral enteritis Acta Vet. Brno., 69 (2019), pp. 116-122, 10.2478/acve-2019-0008

View Record in Scopus Google Scholar

H. Schaefer, B. Kohn, F.J. Schweigert, J. Raila

Quantitative and qualitative urine protein excretion in dogs with severe inflammatory response syndrome

J. Vet. Intern. Med., 25 (2011), pp. 1292-1297, 10.1111/i.1939-1676.2011.00829.x

CrossRefView Record in ScopusGoogle Scholar

S.L. Vaden, C.A. Turman, T.L. Harris, S.L. Marks

The prevalence of albuminuria in dogs and cats in an ICU or recovering from anesthesia

J. Vet. Emerg. Crit. Care, 20 (2010), pp. 479-487, 10.1111/j.1476-4431.2010.00584.x

CrossRefView Record in ScopusGoogle Scholar

Y.H. Lien, T.Y. Hsiang, H.P. Huang

Associations among systemic blood pressure, microalbuminuria and albuminuria in dogs affected with pituitary- and adrenal-dependent hyperadrenocorticism

Acta Vet. Scand., 52 (2010), p. 61, 10.1186/1751-0147-52-61

View Record in ScopusGoogle Scholar

J. Whittemore, B. Marcum, D. Mawby, M. Coleman, T. Hacket, L. Lappin

Associations among albuminuria, C-reactive protein concentrations, survival predictor index scores, and survival in $\mathbf{7 8}$ critically ill dogs

J. Vet. Intern. Med., 25 (2011), pp. 818-824 


\section{CrossRefView Record in ScopusGoogle Scholar}

R. Troìa, M. Gruarin, C. Grisetti, F. Serafini, L. Magna, E. Monari, M. Giunti, F. Dondi

Fractional excretion of electrolytes in volume-responsive and intrinsic acute kidney injury in dogs: diagnostic and prognostic implications

J. Vet. Intern. Med., 32 (2018), pp. 1372-1382, 10.1111/jvim.15146

CrossRefView Record in ScopusGoogle Scholar

M.R. Viant, C. Ludwig, S. Rhodes, U.L. Günther, D. Allaway

Validation of a urine metabolome fingerprint in dog for phenotypic classification

Metabolomics., 3 (2007), pp. 453-463, 10.1007/s11306-007-0092-0

CrossRefView Record in ScopusGoogle Scholar

G. Carlos, F.P. dos Santos, P.E. Fröehlich

Canine metabolomics advances

Metabolomics., 16 (2020), p. 16, 10.1007/s11306-020-1638-7

View Record in ScopusGoogle Scholar

G.M. Forster, J. Stockman, N. Noyes, A.L. Heuberger, C.D. Broeckling, C.M. Bantle, E.P. Ryan

A comparative study of serum biochemistry, metabolome and microbiome parameters of clinically healthy, normal weight, overweight, and obese companion dogs

Top. Companion Anim. Med., 33 (2018), pp. 126-135, 10.1053/j.tcam.2018.08.003

ArticleDownload PDFView Record in ScopusGoogle Scholar

D. Jappar, Y. Hu, R.F. Keep, D.E. Smith

Transport mechanisms of carnosine in SKPT cells: contribution of apical and basolateral membrane transporters

Pharm. Res., 26 (2009), pp. 172-181, 10.1007/s11095-008-9726-9

CrossRefView Record in ScopusGoogle Scholar 
V. Peters, C.Q.F. Klessens, H.J. Baelde, B. Singler, K.A.M. Veraar, A. Zutinic, J. Drozak, J. Zschocke, C.P. Schmitt, E. De Heer

Intrinsic carnosine metabolism in the human kidney

Amino Acids, 47 (2015), pp. 2541-2550, 10.1007/s00726-015-2045-7

CrossRefView Record in ScopusGoogle Scholar

K. Kilis-Pstrusinska

Carnosine and kidney diseases: what we currently know?

Curr. Med. Chem., 20 (2020), 10.2174/0929867326666190730130024

$\underline{\text { Google Scholar }}$

M. Urpi-Sarda, E. Almanza-Aguilera, R. Llorach, R. Vázquez-

Fresno, R. Estruch, D. Corella, J.V. Sorli, F. Carmona, A. Sanchez-Pla, J. Salas-Salvadó, C. AndresLacueva

Non-targeted metabolomic biomarkers and metabotypes of type 2 diabetes: A cross-sectional study of PREDIMED trial participants

Diabetes Metab., 45 (2019), pp. 167-174, 10.1016/j.diabet.2018.02.006

ArticleDownload PDFView Record in ScopusGoogle Scholar

C.J. Chen, W.L. Liao, C.T. Chang, Y.N. Lin, F.J. Tsai

Identification of urinary metabolite biomarkers of type $\mathbf{2}$ diabetes nephropathy using an untargeted metabolomic approach

J. Proteome Res., 17 (2018), pp. 3997-4007, 10.1021/acs.jproteome.8b00644

CrossRefView Record in ScopusGoogle Scholar

D.Q. Chen, G. Cao, H. Chen, D. Liu, W. Su, X.Y. Yu, N.D. Vaziri, X.H. Liu, X. Bai, L. Zhang, Y.Y. Zhao

Gene and protein expressions and metabolomics exhibit activated redox signaling and wnt/ $\beta$ catenin pathway are associated with metabolite dysfunction in patients with chronic kidney disease

Redox Biol., 12 (2017), pp. 505-521, 10.1016/i.redox.2017.03.017

ArticleDownload PDFView Record in ScopusGoogle Scholar 
R.E. Silva, J.L. Baldim, D.A. Chagas-Paula, M.G. Soares, J.H.G. Lago, R.V. Gonçalves, R.D. Novaes

Predictive metabolomic signatures of end-stage renal disease: A multivariate analysis of population-based data

Biochimie., 152 (2018), pp. 14-30, 10.1016/j.biochi.2018.06.009

ArticleDownload PDFView Record in ScopusGoogle Scholar

C.M. Rivara, C.R. Johnson, J.P. Lulich, C.A. Osborne, M. Murtaugh

The effect of disease on the urinary purine metabolite concentrations in dogs

Vet. Rec., 173 (2013), p. 219, 10.1136/vr.101237

CrossRefView Record in ScopusGoogle Scholar

F. Duranton, G. Cohen, R. De Smet, M. Rodriguez, J. Jankowski, R. Vanholder, A. Argiles

Normal and pathologic concentrations of uremic toxins

J. Am. Soc. Nephrol., 23 (2012), pp. 1258-1270, 10.1681/ASN.2011121175

View Record in ScopusGoogle Scholar

M.A. Risso, S. Sallustio, V. Sueiro, V. Bertoni, H. Gonzalez-Torres, C.G. Musso

The importance of tubular function in chronic kidney disease

Int. J. Nephrol. Renovasc. Dis., 12 (2019), pp. 257-262, 10.2147/IJNRD.S216673

CrossRefView Record in ScopusGoogle Scholar

K. lenaga, K. Nakamura, T. Fujisawa, Y. Fukunaga, H. Nihei, M. Narita, Y. Tomino, T. Sanaka, K. Ao yagi, K. Nakano, H. Koide

Urinary excretion of creatol, an in vivo biomarker of hydroxyl radical, in patients with chronic renal failure

Ren. Fail., 29 (2007), pp. 279-283, 10.1080/08860220701219863

CrossRefView Record in ScopusGoogle Scholar

N. Neirynck, R. Vanholder, E. Schepers, S. Eloot, A. Pletinck, G. Glorieux

An update on uremic toxins

Int. Urol. Nephrol., 45 (2013), pp. 139-150, 10.1007/s11255-012-0258-1 


\section{CrossRefView Record in ScopusGoogle Scholar}

A.M. Bosco, B.F.M. Almeida, P.P. Pereira, D.B. dos Santos, J.S. Neto, W.L. Ferreira, P.C. Ciarlini

The uremic toxin methylguanidine increases the oxidative metabolism and accelerates the apoptosis of canine neutrophils

Vet. Immunol. Immunopathol., 185 (2017), pp. 14-19, 10.1016/j.vetimm.2017.01.006

ArticleDownload PDFView Record in ScopusGoogle Scholar

E.P. Rhee, C.B. Clish, A. Ghorbani, M.G. Larson, S. Elmariah, E. McCabe, Q. Yang, S. Cheng, K. Pier ce, A. Deik, A.L. Souza, L. Farrell, C. Domos, R.W. Yeh, I. Palacios, K. Rosenfield, R.S. Vasan, J.C. FI orez, T.J. Wang, C.S. Fox, R.E. Gerszten

A combined epidemiologic and metabolomic approach improves CKD prediction

J. Am. Soc. Nephrol., 24 (2013), pp. 1330-1338, 10.1681/ASN.2012101006

View Record in ScopusGoogle Scholar

F. Aregger, D.E. Uehlinger, G. Fusch, A. Bahonjic, R. Pschowski, M. Walter, J.C. Schefold

Increased urinary excretion of kynurenic acid is associated with non-recovery from acute kidney injury in critically ill patients

BMC Nephrol., 19 (2018), p. 44, 10.1186/s12882-018-0841-5

View Record in ScopusGoogle Scholar

C. Barrios, T.D. Spector, C. Menni

Blood, urine and faecal metabolite profiles in the study of adult renal disease

Arch. Biochem. Biophys., 589 (2016), pp. 81-92, 10.1016/i.abb.2015.10.006

ArticleDownload PDFView Record in ScopusGoogle Scholar

A.L. Saucedo, M.M. Perales-Quintana, D. Paniagua-Vega, C. Sanchez-Martinez, P. CorderoPerez, N.W. Minsky

Chronic kidney disease and the search for new biomarkers for early diagnosis

Curr. Med. Chem., 25 (2018), pp. 3719-3747, 10.2174/0929867325666180307110908

CrossRefView Record in ScopusGoogle Scholar 
T. Chihanga, Q. Ma, J.D. Nicholson, H.N. Ruby, R.E. Edelmann, P. Devarajan, M.A. Kennedy

NMR spectroscopy and electron microscopy identification of metabolic and ultrastructural changes to the kidney following ischemia-reperfusion injury

Am. J. Physiol. Physiol., 314 (2018), pp. 154-166, 10.1152/ajprenal.00363.2017

\section{$\underline{\text { Google Scholar }}$}

M.A. Hanifa, M. Skott, R.G. Maltesen, B.S. Rasmussen, S. Nielsen, J. Frøkiær, T. Ring, R. Wimmer

Tissue, urine and blood metabolite signatures of chronic kidney disease in the 5/6

nephrectomy rat model

Metabolomics., 15 (2019), p. 112, 10.1007/s11306-019-1569-3

View Record in Scopus Google Scholar

A.J. Won, S. Kim, Y.G. Kim, K.B. Kim, W.S. Choi, S. Kacew, K.S. Kim, J.H. Jung, B.M. Lee, S. Kim, H.S . Kim

Discovery of urinary metabolomic biomarkers for early detection of acute kidney injury Mol. BioSyst., 12 (2016), pp. 133-144, 10.1039/c5mb00492f

View Record in ScopusGoogle Scholar

Y. He, W. Su, T. Chen, X. Zeng, Z. Yan, J. Guo, W. Yang, H. Wu

Identification of prototype compounds and derived metabolites of naoxintong capsule in beagle dog urine and feces by UFLC-Q-TOF-MS/MS

J. Pharm. Biomed. Anal., 176 (2019), p. 112806, 10.1016/i.jpba.2019.112806

$\underline{\text { ArticleDownload PDFView Record in ScopusGoogle Scholar }}$

S.M. Titan, G. Venturini, K. Padilha, G. Tavares, R. Zatz, I. Bensenor, P.A. Lotufo, E.P. Rhee, R.I. Th adhani, A.C. Pereira

Metabolites related to eGFR: evaluation of candidate molecules for GFR estimation using untargeted metabolomics

Clin. Chim. Acta, 489 (2019), pp. 242-248, 10.1016/j.cca.2018.08.037

$\underline{\text { ArticleDownload PDFView Record in ScopusGoogle Scholar }}$ 
Y. Wang, D. Lawler, B. Larson, Z. Ramadan, S. Kochhar, E. Holmes, J.K. Nicholson Metabonomic investigations of aging and caloric restriction in a life-long dog study J. Proteome Res., 6 (2007), pp. 1846-1854, 10.1021/pr060685n 